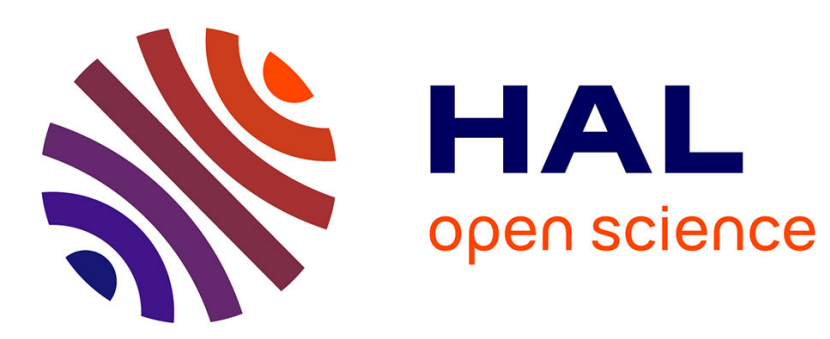

\title{
On the Boyd-Kadomstev System for a three-wave coupling problem and its asymptotic limit
}

\author{
Guy Metivier, Remi Sentis
}

\section{To cite this version:}

Guy Metivier, Remi Sentis. On the Boyd-Kadomstev System for a three-wave coupling problem and its asymptotic limit. 2010. hal-00547084

\section{HAL Id: hal-00547084 \\ https://hal.science/hal-00547084}

Preprint submitted on 15 Dec 2010

HAL is a multi-disciplinary open access archive for the deposit and dissemination of scientific research documents, whether they are published or not. The documents may come from teaching and research institutions in France or abroad, or from public or private research centers.
L'archive ouverte pluridisciplinaire HAL, est destinée au dépôt et à la diffusion de documents scientifiques de niveau recherche, publiés ou non, émanant des établissements d'enseignement et de recherche français ou étrangers, des laboratoires publics ou privés. 


\title{
On the Boyd-Kadomstev System for a three-wave coupling problem and its asymptotic limit.
}

\author{
Guy MÉtivier ${ }^{1}, \quad$ Rémi Sentis ${ }^{2}$
}

April 19, 2010.

Accepted for publication in Communications in Math. Physics

\begin{abstract}
We consider the Boyd-Kadomstev system which is in particular a model for the Brillouin backscattering in laser-plasma interaction. It couples the propagation of two laser beams, the incoming and the backscattered waves, with an ion acoustic wave which propagates at a much slower speed. The ratio $\varepsilon$ between the plasma sound velocity and the (group) velocity of light is small, with typical value of order $10^{-3}$. In this paper, we make a rigorous analysis of the behavior of solutions as $\varepsilon \rightarrow 0$. This problem can be cast in the general framework of fast singular limits for hyperbolic systems. The main new point which is addressed in our analysis is that the singular relaxation term present in the equation is a nonlinear first order system.
\end{abstract}

Keywords : Fast singular Limit, Nonlinear Hyperbolic System, Three-wave Coupling, Laser-plasma Interaction, Brillouin instability, Weak Plasma Turbulence, Boyd-Kadomstev System

\section{Introduction}

We are concerned with the following non linear hyperbolic system

$$
\left\{\begin{array}{l}
\varepsilon \partial_{t} u+\partial_{x} u=-w v \\
\varepsilon \partial_{t} v-\partial_{x} v=\bar{w} u \\
\partial_{t} w+\partial_{x} w=u \bar{v}
\end{array}\right.
$$

on a one-dimension spatial domain $[0, L]$, with initial data

$$
u(0, x)=u_{0}(x), \quad v(0, x)=0, \quad w(0, x)=w_{0}(x),
$$

and boundary data

$$
u(t, 0)=u^{i n}(t), \quad v(t, L)=0, \quad w(t, 0)=0,
$$

\footnotetext{
${ }^{1}$ IMB, Université de Bordeaux, 33405 Talence cedex, France; Guy.Metivier@math.u-bordeaux.fr

${ }^{2}$ CEA, DAM, DIF Bruyères, 91297 Arpajon, France; remi.sentis@cea.fr
} 
This system, called Boyd-Kadomtsev system, is addressed for a long time to model the wave interaction in plasmas. It was first introduced to describe weak plasma turbulence (cf. [9] and [3]) and next used in the framework of laser-plasma interaction. In that case the three complex functions $u=u(t, x), v=v(t, x)$ and $w=w(t, x)$ correspond to the space-time envelope of the main laser wave, the backscattered laser wave due to Brillouin instability and the ion acoustic wave respectively. The boundary value $u^{i n}(t)$ corresponds to the incoming laser field. In this framework, the observation time scale $T$ has the same order of magnitude than the characteristic time of variation of the incoming laser energy $\left|u^{i n}(t)\right|^{2}$. This means that the incoming energy is a smooth function with respect to time $t$ and we may carry out the analysis assuming that $u^{i n}$ and $\partial_{t} u^{i n}$ are bounded functions. On the other hand, the parameter $\varepsilon$ corresponds to the ratio between the plasma sound velocity and the (group) velocity of light. It is assumed to be very small with respect to 1 , its typical value in applications is in the order of $10^{-3}$. The main subject of this paper is to make a detailed analysis of the behavior of the solutions of (1.1) (1.2) (1.3) as $\varepsilon$ tends to 0 .

Of course when dealing with realistic simulations one has to address three-dimension geometry and to account for diffraction, refraction phenomena as well as macroscopic hydrodynamic effects (see [12], [16], [18], [2] for such models). We recall in appendix a glance of the derivation of the models which are handled for such simulations (see also [20], [4], [5], [6] for mathematical justifications in the framework of the Brillouin instability or of the Raman instability and [7], [8], [14] for systematic and rigorous derivation of geometric optics models). For example in the $H E R A$ code (cf. [13]) or the PF3D code (cf. [2]), the modeling is based on a system of the following type

$$
\left\{\begin{array}{c}
\varepsilon \partial_{t} u+\partial_{x} u+i \alpha \Delta_{\perp} u=-\Gamma w v+i \beta_{0}(1-\Gamma) u \\
\varepsilon \partial_{t} v-\partial_{x} v+i \alpha \Delta_{\perp} v=\Gamma \bar{w} u+i \beta_{0}(1-\Gamma) v \\
\partial_{t} w+\partial_{x} w+(\eta+i \omega) w=\Gamma u \bar{v}-w \partial_{x} \log \Gamma
\end{array}\right.
$$

where $\Delta_{\perp}$ is the Laplace operator in the direction transverse to $x$ and where $\alpha, \omega, \eta$ and $\beta_{0}$ are real constants and $\Gamma$ a smooth real function which is close to 1 and related to the macroscopic variation of the electron density. Moreover, in realistic models, the initial value of the ion acoustic wave is a small random noise; here we assume that this initial value is a known function $w_{0}$ which does not depend on the parameter $\varepsilon$. It may be a crude approximation, nevertheless it is a first stage in order to understand the mathematical structure of the problem and to give ideas for efficient numerical schemes for solving system (1.4).

Therefore, we mean the Boyd-Kadomtsev system is sufficient to exhibit most of the difficulties of the three-wave coupling phenomena. For this system, notice first that if $w_{0}=0$, we get a trivial solution which is $v=w=0$ and $u$ solution of the simple advection equation $\varepsilon \partial_{t} u+\partial_{x} u=0$. This trivial solution is of course unstable; this fact is related to the Brillouin instability.

Notice that the following balance relations are crucial for expressing the physical energy conservation:

$$
\left\{\begin{aligned}
\text { i) } & \varepsilon \partial_{t}\left(|u|^{2}+|v|^{2}\right)+\partial_{x}\left(|u|^{2}-|v|^{2}\right)=0 \\
\text { ii) } & \partial_{t}\left(|w|^{2}+\varepsilon|u|^{2}\right)+\partial_{x}\left(|w|^{2}+|u|^{2}\right)=0 \\
\text { iii }) & \partial_{t}\left(|w|^{2}-\varepsilon|v|^{2}\right)+\partial_{x}\left(|w|^{2}+|v|^{2}\right)=0
\end{aligned}\right.
$$

Up to our knowledge, except for the work [17] on solitons (on the full real line), there is no convincing published mathematical work related to this system.

We first show that for a fixed value of $\varepsilon$ (satisfying $0<\varepsilon<1$ ), this semi-linear hyperbolic initialboundary value problem is well-posed in $L^{2}$ and in $L^{\infty}$ (see theorem 2.1). 
After proving this result, one checks that the backscattered energy $|v(t, 0)|^{2}$ is such that $\varepsilon|v(t, 0)|^{2}$ is bounded by a constant (depending on the final time $T$ ). But from a physical point of view, it is natural that the backscattered energy cannot be larger than a constant in the order of the incoming energy. In order to get such a bound, we are led to study the natural asymptotic problem obtained by setting $\varepsilon$ equal to zero, which corresponds to an infinite speed of light.

This limiting system reads as

$$
\left\{\begin{array}{cc}
\partial_{x} u_{*}=-w_{*} v_{*}, & u_{*}(t, 0)=u^{i n}(t), \\
-\partial_{x} v_{*}=\overline{w_{*}} u_{*}, & v_{*}(t, L)=0
\end{array}\right.
$$

coupled with

$$
\partial_{t} w_{*}+\partial_{x} w_{*}=u_{*} \overline{v_{*}} \quad w_{*}(t, 0)=0
$$

and an initial condition on $w$ only

$$
w_{*}(0, x)=w_{0}(x)
$$

We will show (cf. Theorem 3.3) that this system is well-posed in $L^{\infty}$ and that the backscattered energy $\left|v_{*}(t, 0)\right|^{2}$ satisfies the natural bound

$$
\left|v_{*}(t, 0)\right|^{2} \leq\left|u^{i n}(t)\right|^{2}
$$

The main objective of the paper is to prove the convergence of the solutions $\left(u^{\varepsilon}, v^{\varepsilon}, w^{\varepsilon}\right)$ of the full system (1.1)(1.2)(1.3) to the solutions of the limiting problem. This is a singular perturbation problem, with quadratic coupling terms.

Notice that, in general, the solution of the limit problem, does not satisfy the initial condition $\left(u_{*}, v_{*}\right)_{\mid t=0}=\left(u_{0}, 0\right)$ at $t=0$; instead $\left(u_{*}, v_{*}\right)_{\mid t=0}$ satisfy

$$
\left\{\begin{array}{cc}
\partial_{x} u_{* \mid t=0}=-w_{0} v_{* \mid t=0}, & -\partial_{x} v_{* \mid t=0}=\bar{w}_{0} u_{* \mid t=0} \\
u_{* \mid t=0}(0)=u_{\mid t=0}^{i n}, & v_{* \mid t=0}(L)=0 .
\end{array}\right.
$$

This indicates that the solution with $\varepsilon>0$ has a small initial layer in the variables $(u, v)$ to match the initial condition (1.3) to the functions $\left(u_{* \mid t=0}, v_{* \mid t=0}\right)$. The main result we want to prove is the following

Theorem [cf. Theorem 4.1] Suppose that the initial data $u_{0}, w_{0}$ are in $H^{1}(0, L)$ and satisfy the corner conditions (2.1). Then the solutions $\left(u^{\varepsilon}, v^{\varepsilon}, w^{\varepsilon}\right)$ of (1.1) (1.2) (1.3) converges in $\left[L^{2}([0, T] \times\right.$ $[0, L])]^{3}$ to $\left(u_{*}, v_{*}, w_{*}\right)$ the solution of $(1.6)(1.7)(1.8)$.

From a physical point of view this result means, that the backscattered laser intensity $\left|v^{\varepsilon}(t, x)\right|^{2}$ of the initial problem may be approximated (after a small initial layer) by the backscattered laser intensity corresponding to an infinite speed of light which satisfies the natural bound (1.9).

Another motivation for this study comes from numerical issues. Indeed, for three-dimension parallel numerical codes dealing with laser-plasma interaction and based on system like (1.4), the time discretization is performed up to now in an explicit way, using a classical upwind difference scheme to solve the propagation equations for $u$ and $v$. This leads to a sub-cycling technique with a time step $\delta t$ constrained by the criterium $\delta t \leq \varepsilon \delta x$; that is to say $\delta t$ is very small if compared to the characteristic time of the Brillouin instability growth and of course of the characteristic time of the macroscopic hydrodynamic evolution which is in the order of $t_{\text {hydro }}=\delta x$. So it would be interesting to propose 
a time implicit method for the solution of the equations for $u$ and $v$, handling the time derivative $\varepsilon \partial_{t} u$ and $\varepsilon \partial_{t} v$ as perturbative terms (as it is done for classical propagation equations without coupling terms $\varepsilon \partial_{t} u+\partial_{x} u+i \alpha \Delta_{\perp} u=i \beta(1-\Gamma) u$, cf. [1] for example). Our analysis validates this approach and we refer to [21] for a proposition of a numerical implementation based on this idea.

The equation (1.1) can be recast in the form

$$
\partial_{t} \mathcal{U}+L_{0}(\mathcal{U})+\frac{1}{\varepsilon} L_{1}(\mathcal{U})=0
$$

with

$$
\mathcal{U}=\left(\begin{array}{c}
u \\
v \\
w
\end{array}\right), \quad L_{0}(\mathcal{U})=\left(\begin{array}{c}
0 \\
0 \\
\partial_{x} w-u \bar{v}
\end{array}\right), \quad L_{1}(\mathcal{U})=\left(\begin{array}{c}
\partial_{x} u+w v \\
-\partial_{x} v-\bar{w} v \\
0
\end{array}\right) .
$$

Written in this form, the problem falls into the category of fast singular limits or relaxation problems, see e.g. $[10,22,15]$ and the references therein. Compared to the mentioned papers, the main new difficulty is that the relaxation term $L_{1}(\mathcal{U})$ is a nonlinear differential system. Using the conservations (1.5), one easily gets uniform bounds for the solutions (see the following section). With them, one can extract subsequences which converge weakly. To prove that the limit satisfy the expected limiting system (1.6), the difficulty is to pass to the weak limit in the quadratic terms. With a bit of compensated compactness, there is no difficulty for the terms $w v$ and $\bar{w} u$. But the term $\bar{v} u$ is highly nontrivial. In addition, the easy first bounds are not sufficient to pass to the limit in the initial conditions, reflecting again the presence of an initial layer.

The heuristic argument for the proof of the main theorem is general to relaxation problems:

- The fast evolution $\partial_{t} \mathcal{U}+\frac{1}{\varepsilon} L_{1}(\mathcal{U})=0$ brings the initial data to a stationary (with respect to the fast time $t / \varepsilon$ ) solution of $L_{1}(\mathcal{U})=0$. After reducing the problem to homogeneous boundary conditions, the main point is to analyze the linear problem

$$
\partial_{s}\left(\begin{array}{l}
u \\
v
\end{array}\right)+\left(\begin{array}{c}
\partial_{x} u+w v \\
-\partial_{x} v-\bar{w} v
\end{array}\right)=0
$$

where $w$ is given and independent of the fast time $s=t / \varepsilon$. One shows that the energy of solutions of this system (with homogeneous boundary conditions) decays exponentially with respect to $s$.

- Next, the decay of energy result is extended to solutions of (1.11) when $w$ is slowly varying with $s$. This proves that the invariant manifold $\mathcal{M}$ of solutions of $L_{1}(\mathcal{U})=0$ is attractive for the fast evolution.

- Using sufficiently good uniform a priori bounds for the solutions, we can use the properties above to prove that the dynamics of solutions of (1.1) is close to the projected dynamic on $\mathcal{M}$, that is (1.6).

Beside the improvement of the numerical treatment of the system (1.4), it will be interesting in the future to analyze this system with an initial value $w_{0}($.$) which is a small stochastic noise and$ perhaps also to address the case where the plasma density $\Gamma$ may depend on both the space and the time variable.

The outline of the paper is the following. First we give a priori estimates for the solutions of the Boyd-Kadomtsev system (1.1) (1.2) (1.3) and we show that it is well-posed. The next section is devoted to prove the existence and uniqueness of solution of the limiting system. In the last one, we prove the main result. In the appendix, we give a phyiscal glance of the derivation of the Brillouin instability model. 


\section{The full system}

In the sequel of the paper, we assume that the corner compatibility conditions holds

$$
u_{0}(0)=u_{\mid t=0}^{i n}, \quad w_{0}(0)=0 .
$$

According to (1.5) we get the basic estimates

$$
\begin{aligned}
\varepsilon \partial_{t}\left(\|u\|_{L_{x}^{2}}^{2}+\|v\|_{L_{x}^{2}}^{2}\right)+\left(|u(t, L)|^{2}+|v(t, 0)|^{2}\right) & =\left|u^{i n}\right|^{2}, \\
\partial_{t}\left(\|w\|_{L_{x}^{2}}^{2}+\varepsilon\|u\|_{L_{x}^{2}}^{2}\right)+\left(|u(t, L)|^{2}+|w(t, L)|^{2}\right) & =\left|u^{i n}\right|^{2}, \\
\partial_{t}\left(\|w\|_{L_{x}^{2}}^{2}-\varepsilon\|v\|_{L_{x}^{2}}^{2}\right)+|w(t, L)|^{2} & =|v(t, 0)|^{2} .
\end{aligned}
$$

Here and in the sequel, we set $L_{x}^{2}=L^{2}(0, L)$ and $L_{x}^{\infty}=L^{\infty}(0, L)$. Let $\Omega=[0, T] \times[0, L]$. We introduce the three velocities $c_{1}=\varepsilon^{-1}, c_{2}=-\varepsilon^{-1}, c_{3}=1$ and define the operators

$$
K_{i}=\partial_{t}+c_{i} \partial_{x}
$$

The first main result is the following

Theorem 2.1. If the data $u_{0}, w_{0}$ are in $L_{x}^{\infty}$ and $u^{i n}$ in $L_{t}^{\infty}$, the initial-boundary value problem $(1.1)(1.2)(1.3)$ has a unique solution $(u, v, w)=\left(u^{\varepsilon}, v^{\varepsilon}, w^{\varepsilon}\right)$ such that $K_{1} u, K_{2} v, K_{3} w$ are in $L^{2}(\Omega)$. Moreover it belongs to $\left[C^{0}\left(0, T ; L_{x}^{2}\right)\right]^{3}$ and $\left(L^{\infty}(\Omega)\right)^{3}$.

The first subsection is devoted to the existence of a solution $\left(u^{\varepsilon}, v^{\varepsilon}, w^{\varepsilon}\right)$ of system (1.1)(1.2)(1.3) in a $L^{2}$ framework. In the second one, assuming that initial data are in $L_{x}^{\infty}$, we show taht the solutions are in $L^{\infty}(\Omega)$. In the last one, with the hypothesis $u_{0}, w_{0} \in H^{1}(0, L)$, we show that the derivatives $\partial_{x} u^{\varepsilon}, \partial_{x} v^{\varepsilon}, \partial_{x} w^{\varepsilon}, \partial_{t} w^{\varepsilon}$ are bounded in $L^{2}(\Omega)$ uniformely with respect to $\varepsilon$ which will be usefull for the asymptotic analysis (cf theo. 2.7).

\subsection{Existence and uniqueness in $L^{2}$ framework.}

Denote $a_{1}=-\varepsilon^{-1}, a_{2}=\varepsilon^{-1}, a_{3}=1$. Let us address the original system with general initial values $\left(u_{0}, v_{0}, w_{0}\right)$ in $\left(L_{x}^{2}\right)^{3}$ and $u^{i n}$ in $L_{t}^{2}$, it reads as

$$
\begin{aligned}
K_{1} u & =a_{1} v w, & u(0, .) & =u_{0}, & & u(t, 0)=u^{i n} \\
K_{2} v & =a_{2} u \bar{w}, & v(0, .) & =v_{0}, & & v(t, L)=0 \\
K_{3} w & =a_{3} \bar{v} u, & w(0, .) & =w_{0}, & & w(t, 0)=0
\end{aligned}
$$

In this subsection, $\varepsilon$ is fixed and the constants may depend on $\varepsilon$. For a final time denoted by $\tau$, let us introduce the following spaces

$$
\mathcal{V}_{1}^{\tau}=\left\{u=u(t, x), \text { s. t. } K_{1} u \in L^{2}\left(0, \tau ; L_{x}^{2}\right), \quad u(0, .) \in L_{x}^{2}, \quad u(., 0) \in L^{2}(0, \tau)\right\},
$$

endowed with the norms

$$
\|u\|_{\mathcal{V}_{1}^{\tau}}=\left\|K_{1} u\right\|_{L^{2}\left(0, \tau ; L_{x}^{2}\right)}+\|u(0, .)\|_{L_{x}^{2}}+\|u(., 0)\|_{L^{2}(0, \tau)}
$$

and the analogous for $\mathcal{V}_{2}^{\tau}$ and $\mathcal{V}_{3}^{\tau}$, with $K_{1}$ replaced by $K_{2}$ and $K_{3}$ (and the corresponding boundary condition in $L$ for $\mathcal{V}_{2}^{\tau}$ ).

We first have the following local existence result. 
Proposition 2.2. Assume that the initial values $\left(u_{0}, v_{0}, w_{0}\right)$ are in $L_{x}^{2}$. Then, for $\tau$ small enough, there is a unique solution $(u, v, w)$ in $\mathcal{V}_{1}^{\tau} \times \mathcal{V}_{2}^{\tau} \times \mathcal{V}_{3}^{\tau}$ of problem (2.3). Moreover $(u, v, w)$ belongs to $\left(C\left(0, \tau ; L_{x}^{2}\right)\right)^{3}$.

The proof is based the following general result inspired by the one stated in $L^{1}$ framework in [23].

Lemma.(Compensated integrability) There exists a constant $C_{0}$ such that for all $\tau$ and for all functions $u, v$ in $\mathcal{V}_{1}^{\tau} \times \mathcal{V}_{2}^{\tau}$ we get

$$
\|u v\|_{L^{2}\left(0, \tau ; L_{x}^{2}\right)}^{2} \leq C_{0}\left[\alpha_{u}+\tau\left\|K_{1} u\right\|_{L^{2}\left(0, \tau ; L_{x}^{2}\right)}^{2}\right]\left[\alpha_{v}+\tau\left\|K_{2} v\right\|_{L^{2}\left(0, \tau ; L_{x}^{2}\right)}^{2}\right]
$$

with $\alpha_{u}=\|u(., 0)\|_{L^{2}(0, \tau)}^{2}+\left\|u_{0}\right\|_{L_{x}^{2}}^{2}$ and $\alpha_{v}=\|v(., L)\|_{L^{2}(0, \tau)}^{2}+\left\|v_{0}\right\|_{L_{x}^{2}}^{2}$.

The same result holds for the other products $u w$ and $v w$ (and for the products $u \bar{v}, \bar{w} v$ ). Of course the constant $C_{0}$ depends on the velocities $c_{i}$ occuring in the operators $K_{i}$, that is to say on $\varepsilon$.

Proof of the lemma. Denote $f=K_{1} u$, we have

$$
u(t, x)=u_{0}\left(x-c_{1} t\right) \mathbf{1}_{x>c_{1} t}+u^{i n}\left(\frac{c_{1} t-x}{c_{1}}\right) \mathbf{1}_{x<c_{1} t}+\int_{0}^{t} f\left(t-s, x-c_{1} s\right) d s
$$

Then we get for all $t<\tau$,

$$
|u(t, x)|^{2} \leq 2\left(\left|u_{0}\left(x-c_{1} t\right)\right| \mathbf{1}_{x>c_{1} t}+\left|u^{i n}\left(\frac{c_{1} t-x}{c_{1}}\right)\right| \mathbf{1}_{x<c_{1} t}\right)^{2}+2 \tau F\left(x-c_{1} t\right),
$$

with $F(y)=\int_{0}^{t}\left|f\left(y+c_{1} s, s\right)\right|^{2} d s$, that is to say $|u(t, x)|^{2} \leq \phi_{u}\left(x-c_{1} t\right)$ where the function $\phi_{u}$ defined on $\left[-c_{1} \tau, L\right]$ is given by

$$
\phi_{u}(\sigma)=2\left|u_{0}(\sigma)\right|^{2} \mathbf{1}_{\sigma>0}+2\left|u^{i n}\left(-\frac{\sigma}{c_{1}}\right)\right|^{2} \mathbf{1}_{\sigma<0}+2 \tau F(\sigma)
$$

Moreover we see that

$$
\left\|\phi_{u}\right\|_{L_{x}^{1}} \leq 2 \alpha_{u}+2 \tau\left\|K_{1} u\right\|_{L^{2}\left(0, \tau, L_{x}^{2}\right)}^{2}, \quad \alpha_{u}=\left\|u_{0}\right\|_{L_{x}^{2}}^{2}+\|u(., 0)\|_{L_{t}^{2}}^{2} .
$$

By the same way, we get $|v(t, x)|^{2} \leq \phi_{v}\left(x-c_{2} t\right)$ with $\phi_{v}$ such that for all $t \leq \tau$

$$
\left\|\phi_{v}\right\|_{L_{x}^{1}} \leq 2 \alpha_{v}+2 \tau\left\|K_{2} v\right\|_{L^{2}\left(0, \tau, L_{x}^{2}\right)}^{2}, \quad \alpha_{u}=\left\|v_{0}\right\|_{L_{x}^{2}}^{2}+\|u(., L)\|_{L_{t}^{2}}^{2} .
$$

Now with the new variables $y=\left(x-c_{1} t\right)$ and $y^{\prime}=\left(x-c_{2} t\right)$ using the fact that $d x d t=\mid c_{1}-$ $\left.c_{2}\right|^{-1} d y d y^{\prime}$, we get

$$
\iint|u(t, x)|^{2}|v(t, x)|^{2} d x d t \leq \iint\left|c_{1}-c_{2}\right|^{-1} \phi_{u}(y) \phi_{v}\left(y^{\prime}\right) d y d y^{\prime} \leq\left|c_{1}-c_{2}\right|^{-1}\left\|\phi_{u}\right\|_{L_{x}^{1}}\left\|\phi_{v}\right\|_{L_{x}^{1}}
$$

and the result follows.

Proof of prop. 2.2. Denote in the sequel $\mathcal{U}=\{u, v, w\}$. Define the space $\mathcal{L}^{2, \tau}=\left(L^{2}\left(0, \tau, L_{x}^{2}\right)\right)^{3}$ endowed by the norm $\|\mathcal{U}\|_{\mathcal{L}^{2, \tau}}=\sup _{i}\left\|\mathcal{U}_{i}\right\|_{L^{2}\left(0, \tau, L_{x}^{2}\right)}$. Denote $\mathcal{F}(\mathcal{U})=\left\{a_{1} v w, a_{2} u \bar{v}, a_{3} u \bar{v}\right\}$ and $\mathcal{K U}=$ $\left\{K_{1} \mathcal{U}_{1}, K_{2} \mathcal{U}_{2}, K_{3} \mathcal{U}_{3}\right\}$

Existence. It is based on a fixed point algorithm. 
Let us denote by $\mathcal{U}^{0}=\left(u^{0}, v^{0}, w^{0}\right)$ the solution of problem (2.3) without the quadratic right hand side terms and define the sequence $\mathcal{U}^{n+1}=\left(u^{n+1}, v^{n+1}, w^{n+1}\right)$ by

$$
\begin{aligned}
\mathcal{K U}^{n+1} & =\mathcal{F}\left(\mathcal{U}^{n}\right) \\
\mathcal{U}^{n+1}(0, .) & =\left\{u_{0}, v_{0}, w_{0}\right\} \\
u^{n+1}(t, 0) & =u^{i n}, \quad v^{n+1}(t, L)=0, \quad w^{n+1}(t, 0)=0 .
\end{aligned}
$$

Now, denote $\mathcal{K U}^{n}=\mathcal{G}^{n}$. For fixed initial and boundary values, $\mathcal{K}^{-1}$ is well defined, then let us address the mapping

$$
\mathcal{G}^{n} \mapsto \mathcal{G}^{n+1}=\mathcal{F}\left(\mathcal{U}^{n}\right)=\mathcal{F}\left(\mathcal{K}^{-1}\left(\mathcal{G}^{n}\right)\right) .
$$

Let us now consider two elements $\mathcal{G}=\left\{g_{1}, g_{2}, g_{3}\right\}$ and $\widehat{\mathcal{G}}=\left\{\widehat{g_{1}}, \widehat{g_{2}}, \widehat{g_{3}}\right\}$ and for fixed initial and boundary values, define $\mathcal{U}$ and $\widehat{\mathcal{U}}$ by $\mathcal{K U}=\mathcal{G}$ and $\mathcal{K} \widehat{\mathcal{U}}=\widehat{\mathcal{G}}$. Set $\mathcal{F}\left(\mathcal{K}^{-1}(\widehat{\mathcal{G}})\right)-\mathcal{F}\left(\mathcal{K}^{-1}(\mathcal{G})\right)=\left\{a_{1} z_{1}, a_{2} z_{2}, a_{3} z_{3}\right\}$. We see that $z_{1}=\widehat{v} \widehat{w}-v w=\widehat{v}(\widehat{w}-w)+w(\widehat{v}-v)$. Since $K_{3}(\widehat{w}-w)=\left(\widehat{g_{3}}-g_{3}\right)$, according to the previous lemma we know that

$$
\|\widehat{v}(\widehat{w}-w)\|_{\mathcal{L}^{2, \tau}}^{2} \leq C_{0}\left(\alpha_{v}+\tau\|\widehat{\mathcal{G}}\|_{\mathcal{L}^{2, \tau}}^{2}\right) \tau\left\|\widehat{g_{3}}-g_{3}\right\|_{\mathcal{L}^{2, \tau}}^{2}
$$

therefore we get

$$
\left\|\mathcal{F}\left(\mathcal{K}^{-1}(\widehat{\mathcal{G}})\right)-\mathcal{F}\left(\mathcal{K}^{-1}(\mathcal{G})\right)\right\|_{\mathcal{L}^{2, \tau}}^{2} \leq C_{0}\left(C_{1}+\tau\|\widehat{\mathcal{G}}\|_{\mathcal{L}^{2, \tau}}^{2}+\tau\|\mathcal{G}\|_{\mathcal{L}^{2, \tau}}^{2} \tau\|\mathcal{G}-\widehat{\mathcal{G}}\|_{\mathcal{L}^{2, \tau}}^{2}\right.
$$

Using this relation with $\mathcal{G}=\mathcal{G}^{n}$ and $\widehat{\mathcal{G}}=0$, we easily check that for $\tau$ small enough, the sequence $q_{n}=\left\|\mathcal{G}^{n}\right\|_{\mathcal{L}^{2, \tau}}^{2}$ is bounded for all $n$ [indeed it implies $q_{n+1} \leq\left\|\mathcal{U}^{0}\right\|_{\mathcal{L}^{2, \tau}}^{2}+C+C \tau^{2} q_{n}^{2}$ and for $\tau$ small enough, this implies that the sequence $q_{n}$ is bounded].

Now inequality (2.4) implies that the mapping $\mathcal{G}^{n} \mapsto \mathcal{G}^{n+1}$ is a strictly contractant mapping for the norm $\|\cdot\|_{\mathcal{L}^{2, \tau}}$ for $\tau$ small enough. Thus the sequence $\mathcal{G}^{n}$ converges to some element $\mathcal{G}_{*}$ in $\mathcal{L}^{2, \tau}$. This implies that $\mathcal{U}^{n}$ converges in $\mathcal{L}^{2, \tau}$ to $\mathcal{U}_{*}$ solution of $\mathcal{K} \mathcal{U}_{*}=\mathcal{G}_{*}$. Moreover, we see that $\mathcal{G}^{n+1}-\mathcal{F}\left(\mathcal{U}_{*}\right)=$ $\mathcal{F}\left(\mathcal{K}^{-1}\left(\mathcal{G}^{n}\right)\right)-\mathcal{F}\left(\mathcal{K}^{-1}\left(\mathcal{G}_{*}\right)\right)$ converges to 0 in $\mathcal{L}^{2, \tau}$ (using once more $(2.4)$ ). Therefore $\mathcal{G}_{*}=\mathcal{F}\left(\mathcal{U}_{*}\right)$ and $\mathcal{U}_{*}$ is solution to $(2.3)$.

Since the right hand sides $\mathcal{F}(\mathcal{U})_{i}$ belong to $L^{2}\left(0, \tau ; L_{x}^{2}\right)$, classical semi-group arguments imply that the solution $\mathcal{U}$ is in $\left(C\left(0, \tau ; L_{x}^{2}\right)\right)^{3}$.

Uniqueness. Assume that there exist two solutions $\mathcal{U}, \widehat{\mathcal{U}}$; they satisfy $\mathcal{K} \mathcal{U}=\mathcal{F}(\mathcal{U})$ and $\mathcal{K} \widehat{\mathcal{U}}=\mathcal{F}(\widehat{\mathcal{U}})$. Then, setting $\widetilde{\mathcal{U}}=\widehat{\mathcal{U}}-\mathcal{U}$, we get

$$
\left|K_{i} \widetilde{\mathcal{U}}_{i}\right| \leq\left|a_{i}\right|\left(\left|\mathcal{U}_{j} \widetilde{\mathcal{U}}_{j^{\prime}}\right|+\left|\widetilde{\mathcal{U}}_{j} \widehat{\mathcal{U}}_{j^{\prime}}\right|\right), \quad \text { with }\left(j, j^{\prime}\right) \neq i
$$

Acording to previous lemma, since the initial and boundary values of $\tilde{\mathcal{U}}$ are zero, we get

$$
\left\|K_{i} \widetilde{\mathcal{U}}_{i}\right\|_{L_{t, x}^{2}}^{2} \leq\left|a_{i}\right| C_{0}\left[\tau\|\mathcal{K} \tilde{\mathcal{U}}\|_{\mathcal{L}^{2, \tau}}^{2}\left(\alpha_{u}+\tau\|\mathcal{K} \mathcal{U}\|_{\mathcal{L}^{2, \tau}}^{2}\right)+\tau\|\mathcal{K} \widetilde{\mathcal{U}}\|_{\mathcal{L}^{2, \tau}}^{2}\left(\alpha_{u}+\tau\|\mathcal{K} \widehat{\mathcal{U}}\|_{\mathcal{L}^{2, \tau}}^{2}\right)\right] .
$$

Thus $\widetilde{\mathcal{U}}=0$ for $\tau$ small enough.

Corollary. If the data $u_{0}, w_{0}$ are in $L_{x}^{2}$ and $u^{\text {in }}$ in $L_{t}^{2}$, the IBV problem (1.1)(1.2)(1.3) admits a unique solution $(u, v, w)$ in $\mathcal{V}_{1}^{T} \times \mathcal{V}_{2}^{T} \times \mathcal{V}_{3}^{T}$, for all $T$. Moreover $(u, v, w)$ belongs to $\left(C\left(0, T ; L_{x}^{2}\right)\right)^{3}$.

Indeed, according to the conservation law $(2.2)$, we see that the solution $(u, v, w)$ defined on a local time interval may be extented up to the final time $\tau=T$.

With the help of proposition 2.3 and of point i) of proposition 2.6 below, this achieves the proof of theorem 2.1. 


\subsection{Estimates in $L^{\infty}$}

We now make a stronger assumption on the initial data: $u_{0}$ and $w_{0}$ belong to $L_{x}^{\infty}$ and $u^{\text {in }}$ belongs to $L_{t}^{\infty}$. Let $(u, v, w)$ the solution in $\left(C\left(0, T ; L_{x}^{2}\right)\right)^{3}$ to the system $(1.1)(1.2)(1.3)$.

Proposition 2.3. There is a constant $C$ independent of $\varepsilon$, such that

$$
\|w\|_{L^{\infty}(\Omega)} \leq C .
$$

For proving this bound, we first look for estimates of $u$ and $v$ along the characteristics of $\partial_{t}+\partial_{x}$. Consider a point $P=(t, x) \in \Omega$. The backward characteristics hit the boundary at points $P_{u}, P_{v}$ and $P_{w}$. From now on, the various constants $C$ do not depend on $\varepsilon$.

Lemma 2.4. For all $t$ such that $0 \leq t \leq T$, there exists a constant $C$ such that

$$
\begin{aligned}
& \int_{\left[P_{w} P\right]}(1-\varepsilon)|u|^{2} d t^{\prime} \leq C \\
& \int_{\left[P_{w} P\right]}(1+\varepsilon)|v|^{2} d t^{\prime} \leq C
\end{aligned}
$$

Proof Define the points $O=(0,0)$ and $L=(0, L)$.

(i) Assume first $\min \{\varepsilon x, \varepsilon(L-x)\} \leq t \leq x$. The balance relation (1.5-ii) is of divergence form in $\Omega$; we can integrate this relation over the quadrangle $O P_{w} P P_{u}$. So, denoting by $\xi$ the linear abscisse on a segment, we get

$$
\int_{\left[P_{w} P\right]}(1-\varepsilon)|u|^{2} d \xi+\int_{\left[P_{u} P\right]}(1-\varepsilon)|w|^{2} d \xi \leq C
$$

where the constant $C$ involves the integral of the initial and bounadry conditions. Integrate the balance relation $(1.5-\mathrm{i})$ on the triangle $P_{w} P_{v} P$ or the quadrangle $P_{w} L P_{v} P$ :

$$
\int_{\left[P_{w} P\right]}(1+\varepsilon)|v|^{2} d \xi+\varepsilon \int_{\left[P_{v} P\right]} 2|u|^{2} d \xi \leq \int_{\left[P_{w} P\right]}(1-\varepsilon)|u|^{2} d \xi+C
$$

(ii) When $t \geq x$, integrate the balance relation (1.5-ii) over the triangle $P_{w} P P_{u}$ and the proof is similar.

(iii) Assume last $t \leq \min \{\varepsilon x, \varepsilon(L-x)\}$, integrate also the balance relation (1.5-ii) over the triangle $P_{w} P P_{u}$.

The result follows from the fact that $d \xi=\sqrt{2} d t$ along $\left[P_{w} P\right]$.

Proof of prop. 2.3. Integrating along the characteristics of $\partial_{t}+\partial_{x}$, we get

$$
w(P)=w_{0}\left(P_{w}\right)+\int_{\left[P_{w} P\right]} u\left(x-t^{\prime}, t-t^{\prime}\right) \bar{v}\left(x-t^{\prime}, t-t^{\prime}\right) d t^{\prime}
$$

thus according to the previous lemma, we get

$$
|w(P)| \leq C
$$

Lemma 2.5. There is $C$ depending only on the $L^{\infty}$ norms of the data, such that

$$
\sup _{x \in[0, L]}\|u(\cdot, x)\|_{L_{t}^{2}} \leq C, \quad \sup _{x \in[0, L]}\|v(\cdot, x)\|_{L_{t}^{2}} \leq C .
$$

In particular, there is another constant $C$ such that

$$
\|u\|_{L^{2}(\Omega)} \leq C, \quad\|v\|_{L^{2}(\Omega)} \leq C
$$


Proof. Repeat the proof of Lemma 2.4 integrating the same conservation laws over the rectangles $\left\{0 \leq t \leq T, 0 \leq x \leq x_{0}\right\}$ and $\left\{0 \leq t \leq T, x_{0} \leq x \leq L\right\}$ respectively to obtain bounds for $\left\|u\left(\cdot, x_{0}\right)\right\|_{L^{2}}^{2}$ and $\left\|w\left(\cdot, x_{0}\right)\right\|_{L^{2}}^{2}$ first, and next of $\left\|v\left(\cdot, x_{0}\right)\right\|_{L^{2}}^{2}$.

The next result improve the estimates above.

Proposition 2.6. The solution of problem $(u, v, w)$ of system (1.1) (1.2) (1.3) is such that

i) $\sqrt{\varepsilon} u$ and $\sqrt{\varepsilon} v$ are uniformly bounded in $L^{\infty}(\Omega)$.

ii) $u$ and $v$ are uniformly bounded in $L^{2}\left(0, T ; L_{x}^{\infty}\right)$, i.e. there is a constant $C$

$$
\int_{0}^{T}\|u(t, \cdot)\|_{L_{x}^{\infty}}^{2} d t \leq C, \quad \int_{0}^{T}\|v(t, \cdot)\|_{L_{x}^{\infty}}^{2} d t \leq C .
$$

Proof. As above, integrating the transport equation $\varepsilon \partial_{t} u+\partial_{x} u=w v$ along the characteristic, we get after setting $A=\max \left(\left\|u^{i n}\right\|_{L^{\infty}},\left\|u_{0}\right\|_{L^{\infty}}\right)$

$$
|u(t, x)| \leq A+\|w\|_{L^{\infty}} \int_{0}^{\inf \left(\frac{t}{\varepsilon}, x\right)}|v(t-\varepsilon s, x-s)| d s \leq A+\|w\|_{L^{\infty}} M(t-\varepsilon x)
$$

where $M$ is defined by

$$
M(\sigma)=\int_{0}^{L} \mathbf{1}_{\Omega}(\sigma+\varepsilon y, y)|v(\sigma+\varepsilon y, y)| d y
$$

Note that $M(\sigma)=0$ for $\sigma \geq T$ and $\sigma \leq-\varepsilon L$. Moreover one may check that $M$ is in $L^{2}$ with norm

$$
\|M\|_{L^{2}(\mathbb{R})} \leq \sqrt{L}\|v\|_{L^{2}(\Omega)}
$$

Similarly, we get

$$
\frac{|v(t, x)|}{\|w\|_{L^{\infty}}} \leq \int_{0}^{\inf \left(\frac{t}{\varepsilon}, L-x\right)}\left|u\left(t-\varepsilon s^{\prime}, x+s^{\prime}\right)\right| d s^{\prime} \leq N(t+\varepsilon x), \quad N(\sigma)=\int_{0}^{L} \mathbf{1}_{\Omega}(\sigma-\varepsilon y, y)|u(\sigma-\varepsilon y, y)| d y .
$$

here also $N$ is supported in $[-\varepsilon L, T]$ and is in $L^{2}(\mathbb{R})$.

From this inequality and (2.8), we get for all $t \leq T$ and $x$ (with $\mathbf{H}$ the Heaviside function)

$$
\begin{aligned}
M(t-\varepsilon x) & \leq \int_{0}^{L} \mathbf{1}_{\Omega}(t-\varepsilon s, x-s)|v(t-\varepsilon s, x-s)| d s \\
& \leq\|w\|_{L^{\infty}} \int_{0}^{L} \int_{0}^{L} \mathbf{H}\left(t-\varepsilon\left(s+s^{\prime}\right)\right)\left|u\left(t-\varepsilon\left(s+s^{\prime}\right), x-s+s^{\prime}\right)\right| d s d s^{\prime} .
\end{aligned}
$$

Using classical changes of variables, we get

$$
\frac{M(t-\varepsilon x)}{\|w\|_{L^{\infty}}} \leq L\left(\int_{0}^{2 L} \int_{0}^{L} \mathbf{H}(t-\varepsilon \xi)|u(t-\varepsilon \xi, y)|^{2} d y d \xi\right)^{1 / 2} \leq L\left(\int_{0}^{t} \int_{0}^{L}|u(t-s, y)|^{2} \frac{d s}{\varepsilon} d y\right)^{1 / 2} .
$$

Now, since $\|u\|_{L^{2}(\Omega)}$ is bounded, we see that $\sqrt{\varepsilon} M$ is bounded uniformly with respect to $\varepsilon$. Analogously $\sqrt{\varepsilon} N$ is also uniformely bounded; that is to say point $i$ ) holds. 
Moreover, using (2.8), we have

$$
\begin{aligned}
\|u(t, .)\|_{L^{\infty}} & \leq A+\|w\|_{L^{\infty}} \sup _{x} \int_{0}^{L}|v(t-\varepsilon x+\varepsilon y, y)| d y \\
& \leq A+\|w\|_{L^{\infty}}^{2} \sup _{x} \int_{0}^{L} \mathbf{1}_{[0, T]}(t-\varepsilon x+2 \varepsilon y) N(t-\varepsilon x+2 \varepsilon y) d y
\end{aligned}
$$

So, to get the point $i i)$, it suffices to show that the function $\phi$ defined by $\phi(t)=\sup _{x} \int_{-x}^{x} N(t-\varepsilon L+$ $\varepsilon z) d z$ is in $L^{2}(0, T)$ (and the analogous for $v$ ). But we have

$$
\phi(t+\varepsilon L)=\sup _{x} \int_{-x}^{x} N(t+\varepsilon z) d z \leq \frac{1}{\varepsilon} \int_{-L \varepsilon}^{L \varepsilon} N(t+s) d s \leq 2 L N^{*}(t) .
$$

where we have introduced the maximal function $N^{*}$ of $N$ :

$$
N^{*}(t)=\max _{\rho} \frac{1}{2 \rho} \int_{-\rho}^{\rho} N(t+s) d s
$$

By classical harmonic analysis (cf. for example [11]) we know that $\left\|N^{*}\right\|_{L^{2}} \leq C_{4}\|N\|_{L^{2}}$, which implies that $\phi$ is in $L^{2}(0, T)$.

\subsection{Estimates for derivatives}

Besides the previous hypothesis, we make stronger assumptions on $u_{0}$ and $w_{0}$.

Theorem 2.7. Assume that $u_{0}$ and $w_{0}$ are in $H^{1}([0, L]), u^{i n} \in H^{1}([0, T])$ and satisfy the corner conditions (2.1). Then the solutions given by Theorem 2.1 belong to $\left[C^{0}\left(0, T ; H^{1}([0, L])\right) \cap\right.$ $\left.C^{1}\left(0, T ; L^{2}([0, L])\right)\right]^{3}$.

Moreover, there are uniform bounds for $\partial_{x} u, \partial_{x} v, \partial_{x} w, \varepsilon \partial_{t} u, \varepsilon \partial_{t} v$ in $L_{x}^{\infty}\left(0, L ; L_{t}^{2}([0, T])\right)$ thus in $L^{2}(\Omega)$. There are also uniform bounds for $\partial_{t} w$ in $L_{x}^{\infty}\left(0, L ; L_{t}^{1}([0, T])\right)$ and in $L_{t}^{1}\left(0, T ; L_{x}^{2}([0, L])\right)$.

Lemma 2.8. The functions $u_{1}=\left(\partial_{x}-\varepsilon \partial_{t}\right) u$ and $w_{1}=\left(\partial_{x}-\varepsilon \partial_{t}\right) w$ are bounded in $L^{\infty}\left(0, L ; L_{t}^{2}\right)$. Moreover, $w_{1}$ is also bounded in $L^{\infty}\left(0, T ; L_{x}^{2}\right)$.

Proof Differentiate the first and third equations with respect to $\partial_{x}-\varepsilon \partial_{t}$ :

$$
\left\{\begin{array}{c}
\varepsilon \partial_{t} u_{1}+\partial_{x} u_{1}=-v w_{1}+|w|^{2} u \\
\partial_{t} w_{1}+\partial_{x} w_{1}=\bar{v} u_{1}-|u|^{2} w
\end{array}\right.
$$

The initial-boundary values which read as

$$
\left\{\begin{array}{c}
u_{1}(0, x)=2 \partial_{x} u(0, x)+w v(0, x)=2 \partial_{x} u_{0}(x) \\
u_{1}(t, 0)=-2 \varepsilon \partial_{t} u^{i n}(t)-w v(t, 0)=-2 \varepsilon \partial_{t} u^{i n}(t) \\
w_{1}(0, x)=(1+\varepsilon) \partial_{x} w_{0}(x)-\varepsilon u \bar{v}(0, x)=(1+\varepsilon) \partial_{x} w_{0}(x) \\
w_{1}(t, 0)=u^{i n}(t) \bar{v}(t, 0)
\end{array}\right.
$$

are bounded in $L_{t}^{2}$ or $L_{x}^{2}$, since we already have a bound of $v_{\mid x=0}$ in $L_{t}^{2}$ by Lemma 2.5 .

The equations imply that

$$
\partial_{t}\left(\varepsilon\left|u_{1}\right|^{2}+\left|w_{1}\right|^{2}\right)+\partial_{x}\left(\left|u_{1}\right|^{2}+\left|w_{1}\right|^{2}\right)=|w|^{2}\left(\partial_{x}-\varepsilon \partial_{t}\right)|u|^{2}-|u|^{2}\left(\partial_{x}-\varepsilon \partial_{t}|w|^{2}\right)
$$


Hence:

$$
\partial_{t}\left(\varepsilon\left|u_{1}\right|^{2}+\left|w_{1}\right|^{2}\right)+\partial_{x}\left(\left|u_{1}\right|^{2}+\left|w_{1}\right|^{2}\right)+\left(\partial_{x}-\varepsilon \partial_{t}\right)\left(|u|^{2}|w|^{2}\right)=4 \operatorname{Re}|w|^{2} u \bar{u}_{1}
$$

Integrate this identity over the quadrangle $O P^{\prime} P Q$, where $P=(t, x), P^{\prime}=(0, x), Q=(t, 0)$ and $O=(0,0)$. Then

$$
\begin{gathered}
\int_{0}^{t}\left(\left|u_{1}\right|^{2}+\left|w_{1}\right|^{2}\right)\left(t^{\prime}, x\right) d t^{\prime}+\int_{0}^{x}\left(\varepsilon\left|u_{1}\right|^{2}+\left|w_{1}\right|^{2}\right)\left(t, x^{\prime}\right) d x^{\prime}+\int_{0}^{t}|u|^{2}|w|^{2}\left(t^{\prime}, x\right) d t^{\prime} \\
+\int_{0}^{x} \varepsilon|u|^{2}|w|^{2}\left(0, x^{\prime}\right) d x^{\prime}=\int_{0}^{t}\left(\left|u_{1}\right|^{2}+\left|w_{1}\right|^{2}\right)\left(t^{\prime}, 0\right) d t^{\prime}+\int_{0}^{x}\left(\varepsilon\left|u_{1}\right|^{2}+\left|w_{1}\right|^{2}\right)\left(0, x^{\prime}\right) d x^{\prime} \\
\quad+\int_{0}^{t}|u|^{2}|w|^{2}\left(t^{\prime}, 0\right) d t^{\prime}+\int_{0}^{x} \varepsilon|u|^{2}|w|^{2}\left(t, x^{\prime}\right) d x^{\prime}+\int 4 \operatorname{Re}|w|^{2} u \bar{u}_{1}\left(t^{\prime}, x^{\prime}\right) d t^{\prime} d x^{\prime}
\end{gathered}
$$

The boundary terms in the right hand side are bounded. Moreover, $w$ is bounded in $L^{\infty}$. Therefore

$$
\int_{0}^{t}\left(\left|u_{1}\right| 2+\left|w_{1}\right|^{2}\right)\left(t^{\prime}, x\right) d t^{\prime}+\int_{0}^{x}\left|w_{1}\right|^{2}\left(t, x^{\prime}\right) d x^{\prime} \leq C+C \varepsilon \int_{0}^{x}\left|u\left(t, x^{\prime}\right)\right|^{2} d x^{\prime}+C \iint|u|\left|u_{1}\right|\left(t^{\prime}, x^{\prime}\right) d t^{\prime} d x^{\prime} .
$$

By Lemma 2.5, the second term in the right hand side is bounded. We already have a bound for $u$ in $L_{x}^{\infty}\left(L_{t}^{2}\right)$, thus we can absorb the double integral from the right to the left using Gronwall's lemma, and conclude that

$$
\int_{0}^{t}\left(\left|u_{1}\right|^{2}+\left|w_{1}\right|^{2}\right)\left(t^{\prime}, x\right) d t^{\prime}+\int_{0}^{x}\left|w_{1}\left(t, x^{\prime}\right)\right|^{2} d x^{\prime} \leq C .
$$

This proves the lemma.

Lemma 2.9. The functions $v_{2}=\left(\partial_{x}+\varepsilon \partial_{t}\right) v$ and $w_{2}=\left(\partial_{x}+\varepsilon \partial_{t}\right) \bar{w}$ are bounded in $L_{x}^{\infty}\left(L_{t}^{2}\right)$, thus in $L^{2}(\Omega)$.

Proof Differentiate the second and third equations with respect to $\partial_{x}+\varepsilon \partial_{t}$ :

$$
\left\{\begin{array}{c}
\left(\partial_{x}-\varepsilon \partial_{t}\right) v_{2}=-u w_{2}+|w|^{2} v \\
\quad\left(\partial_{x}+\partial_{t}\right) w_{2}=\bar{u} v_{2}-|v|^{2} \bar{w}
\end{array}\right.
$$

The initial-boundary values wich read as

$$
\left\{\begin{array}{c}
v_{2}(0, x)=2 \partial_{x} v(0, x)+\bar{w} u(0, x)=\overline{w_{0}} u_{0} \\
v_{2}(t, L)=2 \varepsilon \partial_{t} v(t, L)-\bar{w} u(t, L) \\
w_{2}(0, x)=(1-\varepsilon) \partial_{x} \overline{w_{0}}(x)+\varepsilon \bar{u} v(0, x) \\
w_{2}(t, L)=(\varepsilon-1) \partial_{t} \bar{w}(t, L)-\bar{u} v(t, L)=\frac{1+\varepsilon}{1-\varepsilon} \bar{w}_{1}(t, L)
\end{array}\right.
$$

are bounded in $L_{t}^{2}$ or $L_{x}^{2}$ since we know that $\left.u\right|_{x=L} \in L_{t}^{2}$ and $\left.w_{1}\right|_{x=L}=\in L_{t}^{2}$.

The equations imply that

$$
\partial_{t}\left(\left|w_{2}\right|^{2}-\varepsilon\left|v_{2}\right|^{2}\right)+\partial_{x}\left(\left|v_{2}\right|^{2}+\left|w_{2}\right|^{2}\right)=|w|^{2}\left(\partial_{x}+\varepsilon \partial_{t}\right)|v|^{2}-|v|^{2}\left(\partial_{x}+\varepsilon \partial_{t}|w|^{2}\right)
$$

Hence:

$$
\partial_{t}\left(\left|w_{2}\right|^{2}-\varepsilon\left|v_{2}\right|^{2}\right)+\partial_{x}\left(\left|v_{2}\right|^{2}+\left|w_{2}\right|^{2}\right)+\left(\partial_{x}+\varepsilon \partial_{t}\right)\left(|v|^{2}|w|^{2}\right)=4 \operatorname{Re}|w|^{2} v \bar{v}_{2}
$$

Given $P=(t, x) \in \Omega$, integrate this identity over the rectangle $P^{\prime} O^{\prime} Q^{\prime} P$, where $P^{\prime}=(0, x)$, $Q^{\prime}=(t, L)$ and $O^{\prime}=(0, L)$. Then 


$$
\begin{aligned}
& \int_{0}^{t}\left(\left|v_{2}\right|^{2}+\left|w_{2}\right|^{2}\right)\left(t^{\prime}, x\right) d t^{\prime}+\int_{x}^{L} \varepsilon\left|v_{2}\left(t, x^{\prime}\right)\right|^{2} d x^{\prime}+\int_{x}^{L}\left|w_{2}\left(0, x^{\prime}\right)\right|^{2} d x^{\prime}+ \\
& +\int_{0}^{t}|v|^{2}|w|^{2}\left(t^{\prime}, x\right) d t^{\prime}+\int_{x}^{L} \varepsilon|v|^{2}|w|^{2}\left(0, x^{\prime}\right) d x^{\prime} \\
= & \int_{0}^{t}\left(\left|v_{2}\right|^{2}+\left|w_{2}\right|^{2}\right)\left(t^{\prime}, L\right) d t^{\prime}+\int_{x}^{L}\left|w_{2}\left(t, x^{\prime}\right)\right|^{2} d x^{\prime}+\int_{x}^{L} \varepsilon\left|v_{2}\left(0, x^{\prime}\right)\right|^{2} d x^{\prime} \\
& +\int_{0}^{t}|v|^{2}|w|^{2}\left(t^{\prime}, L\right) d t^{\prime}+\int_{x}^{L} \varepsilon|v|^{2}|w|^{2}\left(t, x^{\prime}\right) d x^{\prime}-\iint 4 \operatorname{Re}|w|^{2} v \bar{v}_{2}\left(t^{\prime}, x^{\prime}\right) d t^{\prime} d x^{\prime}
\end{aligned}
$$

In the right hand side, the first and third boundary integrals are controlled by (2.13). The fourth one, also a boundary integral, vanishes, while the fifth is bounded since we know that $w$ is bounded in $L^{\infty}$ and that $\sqrt{\varepsilon} v(t, \cdot)$ is bounded in $L^{2}$. Moreover,

$$
w_{2}=\frac{1-\varepsilon}{1+\varepsilon} \bar{w}_{1}+\frac{2 \varepsilon}{1+\varepsilon} \bar{u} v
$$

Moreover, by Lemma 2.8 we have a bound for $\bar{w}_{1}(t, \cdot)$ in $L^{2}$, and by Lemma 2.5 Proposition 2.6 we have a uniform bound for the $L^{2}$ norm of $\varepsilon u v(t, \cdot)$. Therefore, the integrals

$$
\int_{x}^{L}\left|w_{2}\left(t, x^{\prime}\right)\right|^{2} d x^{\prime}
$$

are uniformly bounded. Therefore, we end up with an inequality of the form

$$
\int_{0}^{t}\left(\left|v_{2}\right|^{2}+\left|w_{2}\right|^{2}\right)\left(t^{\prime}, x\right) d t^{\prime} \leq C+M \int_{0}^{t} \int_{x}^{L}|v|\left|v_{2}\right|\left(t^{\prime}, x^{\prime}\right) d t^{\prime} d x^{\prime}
$$

We already have a bound for $v$ in $L_{x}^{\infty}\left(L_{t}^{2}\right)$. We conclude using Gronwall's lemma.

\section{The limiting problem}

When $w_{*}$ is given, the problem (1.6) is a linear one, thus to deal with it we may assume that the boundary value $u^{i n}$ is set to 1 . So, for a fixed function $W \in L_{x}^{\infty}$, we first address the following stationary system on $[0, L]$

$$
\partial_{x} u+W v=0, \quad \partial_{x} v+\bar{W} u=0
$$

with the boundary conditions

$$
u(0)=1, \quad v(L)=0 .
$$

Proposition 3.1. For all $W \in L_{x}^{\infty}$, the problem (3.1) (3.2) has a unique solution $(u, v)=\mathcal{Y}(W)$ in $\left[L_{x}^{\infty}\right]^{2}$. It satisfies

$$
\begin{gathered}
\|u\|_{L_{x}^{\infty}} \leq \exp \left(\sqrt{L}\|W\|_{L_{x}^{2}}\right), \quad\|v\|_{L_{x}^{\infty}} \leq \exp \left(\sqrt{L}\|W\|_{L_{x}^{2}}\right) \\
|v(t, 0)| \leq 1
\end{gathered}
$$


Moreover, for all $w_{\infty}>0$, there exists $C\left(w_{\infty}\right)$ such that for two functions $W, \widetilde{W}$ whose norms in $L_{x}^{\infty}$ are smaller than $w_{\infty}$, we have

$$
\|\mathcal{Y}(W)-\mathcal{Y}(\widetilde{W})\|_{\left[L_{x}^{\infty}\right]^{2}} \leq C\left(w_{\infty}\right)\|W-\widetilde{W}\|_{L_{x}^{2}}
$$

Before proving this result, notice first that

$$
\partial_{x}\left(|u(x)|^{2}-|v(x)|^{2}\right)=0
$$

so that there is a conserved quantity

$$
\mu=|u(x)|^{2}-|v(x)|^{2} .
$$

From the boundary values, we get that: $0 \leq \mu \leq 1$

Let us define now $z=u \bar{v}$, it satisfies $\partial_{x} z+W\left(|u|^{2}+|v|^{2}\right)=0$. Using that $\left(|u|^{2}+|v|^{2}\right)^{2}=$ $\mu^{2}+4|u|^{2}|v|^{2}=\mu^{2}+4|z|^{2}$, we see that $z$ solves the backward ordinary differential equation

$$
-\partial_{x} z=W \sqrt{\mu^{2}+4|z|^{2}}, \quad z(L)=0 .
$$

Thus $z=\mu \zeta$, where $\zeta$ is the unique solution of

$$
-\partial_{x} \zeta=W \sqrt{1+4|\zeta|^{2}}, \quad \zeta(L)=0 .
$$

The end value at 0 implies

$$
|z(0)|^{2}=\mu^{2}|\zeta(0)|^{2}=1-\mu
$$

Therefore, necessarily

$$
\mu=\frac{\sqrt{1+4|\zeta(0)|^{2}}-1}{2|\zeta(0)|^{2}} \in[0,1]
$$

When $\zeta, \mu$ are known, we get $|u|^{2}=\frac{\mu}{2}\left(\sqrt{1+4|\zeta|^{2}}+1\right)$ and $|v|^{2}=\frac{\mu}{2}\left(\sqrt{1+4|\zeta|^{2}}-1\right)$ and $u$ solves

$$
\partial_{x} u=-W a u, \quad u(0)=1, \quad \text { whith } \quad a=\frac{|v|^{2}}{z}=\frac{\sqrt{1+4|\zeta|^{2}}-1}{2 \zeta},
$$

Therefore to get a solution of (3.1) (3.2), it suffices to use 3 steps:

1) let $\zeta$ be the unique solution of (3.8);

2 ) then $\mu$ is given by (3.10) and $z=\mu \zeta$;

3) then one solves (3.11) to determine $u$ and $a$.

Lastly, we check that $u, v=a u$ is a solution; indeed we have $v(L)=0$ and

$$
-\partial_{x} v=W a^{2} u+u \partial_{x} \zeta\left(\frac{\bar{\zeta}}{\zeta \sqrt{1+4|\zeta|^{2}}}-\frac{\sqrt{1+4|\zeta|^{2}}-1}{2 \zeta^{2}}\right)+\frac{u \partial_{x} \bar{\zeta}}{\sqrt{1+4|\zeta|^{2}}}=\bar{W} u
$$

Now, if $(u, v)$ is a solution to system (3.1) (3.2) with a given function $W$, we denote

$$
\Lambda(W)=z=u \bar{v}
$$

We now review the different steps to study the dependence of the solution on $W$. 
Lemma 3.2. For $W \in L_{x}^{\infty}$, the o.d.e. (3.8) has a unique solution $\zeta$ in $W^{1, \infty}([0, L])$ satisfying

$$
\|\zeta\|_{L^{\infty}} \leq \frac{1}{2} e^{2\|W\|_{L^{1}}}
$$

Moreover, for two functions $W, \widetilde{W}$ whose $L_{x}^{\infty}$ norms are smaller than $w_{\infty}$, if $\zeta$ and $\widetilde{\zeta}$ are the corresponding solutions of (3.8), we get

$$
\begin{aligned}
\|\zeta-\widetilde{\zeta}\|_{L^{\infty}} & \leq C\left(w_{\infty}\right)\|W-\widetilde{W}\|_{L^{2}} . \\
\|\Lambda(W)-\Lambda(\widetilde{W})\|_{L^{\infty}} & \leq C\left(w_{\infty}\right)\|W-\widetilde{W}\|_{L^{2}} .
\end{aligned}
$$

\section{Proof}

Since the function $\zeta \mapsto K(\zeta)=\sqrt{1+4|\zeta|^{2}}$ is globally Lipschitz on $\mathbb{C}$, the o.d.e has a unique solution $\zeta$ defined on the whole interval $[0, L]$. Moreover, since $-\partial_{x} K(\zeta)=-4 \operatorname{Re}(\bar{W} \zeta) \leq 4|W||\zeta| \leq 2|W| K(\zeta)$,

$$
2|\zeta(x)| \leq K(\zeta(x)) \leq e^{2\|W\|_{L^{1}}} .
$$

implying (3.12). This defines the mapping $W \mapsto \zeta$ from $L^{1}$ to $W^{1,1}$ and if $W \in L^{\infty}$, it is clear that $\zeta \in W^{1, \infty}$.

Let $\xi=\widetilde{\zeta}-\zeta$ and $\chi=\widetilde{W}-W$. One has

$$
-\partial_{x} \xi=\widetilde{W}\left(\sqrt{4|\zeta+\xi|^{2}+1}-\sqrt{4|\zeta|^{2}+1}\right)+\chi K(\zeta),
$$

implying that

$$
-\partial_{x}|\xi|^{2} \leq 8|\xi|^{2} w_{\infty}+8|\xi||\chi| e^{2 L w_{\infty}}, \quad \xi(L)=0
$$

hence that

$$
|\xi(x)| \leq e^{2 L w_{\infty}} \int_{x}^{L} e^{4(x-s) w_{\infty}}|\chi(s)| d s \leq C_{0}\left(w_{\infty}\right)\|\chi\|_{L^{2}} .
$$

implying (3.13)

Now, the mapping $\Phi(s)=(\sqrt{1+4 s}-1) / 2 s$ extend as a smooth function near $s=0$, with $\Phi(0)=1$ and it is uniformly Lipschitz. Therefore, since $\mu(W)=\Phi\left(|\zeta(0)|^{2}\right)$ and $\mu(\widetilde{W})=\Phi\left(|\widetilde{\zeta(0)}|^{2}\right)$, this Lipschitz continuity implies using (3.13) that

$$
|\mu(\widetilde{W})-\mu(W)| \leq C_{1}\left(w_{\infty}\right)\|\widetilde{W}-W\|_{L^{2}} .
$$

This bound and (3.13) implies the last result of the lemma.

\section{Proof of Proposition 3.1}

If $\tilde{a}$ and $\tilde{u}$ are associated to $\widetilde{W}$, there holds

$$
-\partial_{x}(\tilde{u}-u)=\widetilde{W} \tilde{a}(\tilde{u}-u)+(\widetilde{W} \tilde{a}-W a) u, \quad(\tilde{u}-u)(0)=0
$$

According to (3.13), we get $\|\widetilde{a}-a\|_{L^{\infty}} \leq C_{1}\left(w_{\infty}\right)\|\widetilde{W}-W\|_{L^{2}}$, then the Lipschitz continuity of $u$ with respect to $W$ follows. This implies immediately the Lipschitz continuity of $v=a u$ and (3.5). Moreover one has

$$
\partial_{x}\left(|u|^{2}+|v|^{2}\right) \leq 4 \operatorname{Re}(z \bar{W}) \leq 2|W|\left(|u|^{2}+|v|^{2}\right) .
$$


Since $\left(|u|^{2}+|v|^{2}\right)(L)=\mu \leq 1$, we get

$$
\log \left(|u|^{2}+|v|^{2}\right) \leq 2 \int_{0}^{L}|W| d x \leq 2 \sqrt{L}\|W\|_{L^{2}}
$$

and (3.3) follows. The bound (3.4) comes from (3.6).

According to the definition of $\Lambda$, the fact that $\left(u_{*}, v_{*}, w_{*}\right)$ is a solution of (1.6) (1.7) (1.8) means that

$$
\left(\partial_{t}+\partial_{x}\right) w_{*}=\left|u^{i n}\right|^{2} \Lambda\left(w_{*}\right), \quad w_{* \mid t=0}=w_{0}, \quad w_{*}(t, 0)=0 .
$$

Thus, it remains to solve (3.16).

Theorem 3.3. For $w_{0}$ in $L_{x}^{\infty}$, the problem (1.6) (1.7) (1.8) has a unique solution $\left(u_{*}, v_{*}, w_{*}\right)$ in $\left[L^{\infty}([0, T] \times[0, L])\right]^{3}$. It satisfies for all $t$

$$
\left|v_{*}(t, 0)\right| \leq\left|u^{i n}(t)\right|
$$

We first prove a local in time existence theorem and next conclude using uniform a priori bounds.

Lemma 3.4. For any $w_{0}$ in $L_{x}^{\infty}$, there exists a time $t_{f}$ depending only on $\left\|w_{0}\right\|_{L^{\infty}},\left\|u^{i n}\right\|_{L^{\infty}}$ and $L$ such that the equation

$$
\left(\partial_{t}+\partial_{x}\right) w=\left|u^{i n}\right|^{2} \Lambda(w),\left.\quad w\right|_{x=0}=0,\left.\quad w\right|_{t=0}=w_{0} .
$$

has a solution $w$ belonging to $L^{\infty}\left(\left[0, t_{f}\right] \times[0, L]\right)$.

Proof Denote $\mathbf{T}_{t}$ the semi-group corresponding to the advection $\left(\partial_{t}+\partial_{x}\right)$ with the homogeneous boundary condition in $x=0$. It is a contraction in $L^{p}$ for all $p$. One solves the equation

$$
W(t)=\mathbf{T}_{t} w_{0}+\mathcal{T}(W)(t) \quad \text { with } \mathcal{T}(W)(t)=\int_{0}^{t} \mathbf{T}_{t-s}\left(\left|u^{i n}\right|^{2} \Lambda(W(s))\right) d s
$$

by Picard's fixed point theorem. By (3.3),

$$
\|\Lambda(W)(t)\|_{L_{x}^{\infty}} \leq \exp \left(2 \sqrt{L}\|W(t)\|_{L_{x}^{2}}\right)\left|u^{i n}\right|,
$$

therefore, if $Y(t)=2 \sqrt{L} \sup _{s<t}\|W(s)\|_{L_{x}^{\infty}}$, we get $\|\mathcal{T}(W)\|_{L_{x}^{2}} \leq t \sqrt{L}\left\|u^{i n}\right\|_{L^{\infty}}^{2} \exp (Y(t))$; and there exists $C$ such that

$$
Y(t) \leq C+2 t L\left\|u^{i n}\right\|_{L^{\infty}}^{2} \exp (Y(t)) .
$$

So for $t$ small enough, $Y(t)$ is bounded and there exists aconstant $w_{\infty}$, such that $\sup _{s<t}\|W(s)\|_{L_{x}^{\infty}} \leq$ $w_{\infty}$. Moreover, Proposition 3.1 implies that for $W$ and $\widetilde{W}$ with $L^{\infty}$ norm bounded by $w_{\infty}$, there is $C\left(w_{\infty}\right)$ such that

$$
\|\mathcal{T}(W)(t)-\mathcal{T}(\widetilde{W})(t)\|_{L_{x}^{\infty}} \leq t\left\|u^{i n}\right\|_{L^{\infty}}^{2} C\left(w_{\infty}\right)\|W-\widetilde{W}\|_{L^{\infty}([0, t] \times[0, L])}
$$

From here, it is clear that if $t_{f}$ is small enough, the mapping $W \mapsto \mathbf{T}_{t} w_{0}+\mathcal{T}(W)(t)$ is Lipschitz continuous from the ball of center $\mathbf{T}_{t} w_{0}$ and radius 1 in $L^{\infty}\left(\left[0, t_{f}\right] \times[0, L]\right)$ into itself, with Lipschitz constant $<1$, implying the existence of a fixed point in this ball. 


\section{Proof of Theorem 3.3. .}

A priori estimates. Assume that there exists a solution $w_{*}$ of (3.16), then multiplying (3.16) by $\bar{w}$ and integrating with respect to the space variable, we have

$$
\partial_{t}\left\|w_{*}(t)\right\|_{L^{2}}^{2}+\left|w_{*}(t, L)\right|^{2}=2 \int_{0}^{L} \operatorname{Re}\left(u_{*} \overline{v_{*} w_{*}}\right) d x
$$

But, integrating (1.6) over $[0, L]$, we get $2 \operatorname{Re} \int\left(u_{*} \overline{v_{*} w_{*}}\right) d x=-\int \partial_{x}\left|u_{*}\right|^{2} \leq\left|u^{i n}\right|^{2}$; so we get

$$
\left\|w_{*}(t)\right\|_{L^{2}} \leq\left\|w_{0}\right\|_{L^{2}}+T\left\|u^{i n}\right\|_{L^{\infty}}^{2}
$$

Next, according to (3.3) we have for all $t \leq T$

$$
\left\|u_{*}(t)\right\|_{L^{\infty}} \leq \exp \left(\sqrt{L}\left(\left\|w_{0}\right\|_{L^{2}}+T\left\|u^{i n}\right\|_{L^{\infty}}^{2}\right)\right), \quad\left\|v_{*}(t)\right\|_{L^{\infty}} \leq \exp \left(\sqrt{L}\left(\left\|w_{0}\right\|_{L^{2}}+T\left\|u^{i n}\right\|_{L^{\infty}}^{2}\right)\right) .
$$

Therefore there exists $w_{\infty}$ depending only on $\left\|w_{0}\right\|_{L^{2}}$ and $L$ such that

$$
\left\|w_{*}(t)\right\|_{L^{\infty}} \leq w_{\infty} .
$$

Uniqueness. Assume that there exist two solutions $w$ and $\widetilde{w}$ satisfying $\|w(t)\|_{L^{\infty}},\|\widetilde{w}(t)\|_{L^{\infty}} \leq w_{\infty}$ and

$$
\left(\partial_{t}+\partial_{x}\right) w=\left|u^{i n}\right|^{2} \Lambda(w), \quad\left(\partial_{t}+\partial_{x}\right) \widetilde{w}=\left|u^{i n}\right|^{2} \Lambda(\widetilde{w}),
$$

with vanishing boundary condition at $x=0$. Using that $(w-\widetilde{w})(t)=\int_{0}^{t} \mathbf{T}_{t-\tau}(\Lambda(\widetilde{w}+\zeta)-\Lambda(\widetilde{w}))(\tau) d \tau$, we get

$$
\left.|(w-\widetilde{w})(t, x)| \leq\left\|u^{i n}\right\|_{L^{\infty}}^{2} \int_{0}^{t} \| \Lambda(w)-\Lambda(\widetilde{w})\right) \|_{L^{\infty}} d \tau
$$

The Lipschitz continuity of $\Lambda$ implies that

$$
\|(w-\widetilde{w})(t)\|_{L^{2}} \leq \sqrt{L} C\left(w_{\infty}\right) \int_{0}^{t}\|(w-\widetilde{w})(s)\|_{L^{2}} d s,
$$

and uniqueness follows from Gronwall's lemma.

Existence. By Lemma 3.4 one knows that for $t_{f}$ small enough, but depending only on the $L^{\infty}$ norms of the data and $L$, there exists a solution $w_{*}(t) \in L^{\infty}\left(\left[0, t_{f}\right] \times[0, L]\right)$. By the a priori bound (3.21), the norm of $w\left(t_{f}\right)$ in $L^{\infty}([0, L])$ is bounded by a constant, which depends only on the data, so that the solution can be continued to $[0, T]$.

The bound of $\left|v_{*}(t)\right|$ comes from (3.4).

\section{$4 \quad$ Asymptotic analysis}

In the sequel we use the following generic notations for the sake of conciseness: $U(t, x)=\left(\begin{array}{c}u(t, x) \\ v(t, x)\end{array}\right)$ whose value is in $\mathbb{C}^{2}$; for $w$ in $\mathbb{C}$ and $U$ in $\mathbb{C}^{2}$. Let $w \perp U$ denote the vector

$$
w \perp U=w \perp\left(\begin{array}{l}
u \\
v
\end{array}\right)=\left(\begin{array}{c}
w v \\
-\bar{w} u
\end{array}\right) .
$$


For all bounded function $w$, consider the operator

$$
M_{w} U=\left(\begin{array}{c}
\partial_{x} u+w v \\
-\partial_{x} v-\bar{w} u
\end{array}\right)
$$

Therefore the original system (1.1) (1.2) (1.3) is equivalent to find $w^{\varepsilon}$ and $U^{\varepsilon}=\left(u^{\varepsilon}, v^{\varepsilon}\right)$ satisfying

$$
\varepsilon \partial_{t} U^{\varepsilon}+M_{w^{\varepsilon}} U^{\varepsilon}=0
$$

with initial and boundary conditions

$$
u_{\mid t=0}^{\varepsilon}=u_{0}, \quad v_{\mid t=0}^{\varepsilon}=0 ; \quad u^{\varepsilon}(t, 0)=u^{i n}, \quad v^{\varepsilon}(t, L)=0 .
$$

and

$$
\partial_{t} w^{\varepsilon}+\partial_{x} w^{\varepsilon}=u^{\varepsilon} \overline{v^{\varepsilon}}, \quad w_{\mid t=0}^{\varepsilon}=w_{0}, \quad w^{\varepsilon}(t, 0)=0 .
$$

The aim of this section is to prove the

Theorem 4.1 (Main result). Suppose that the initial data $u_{0}, w_{0}$ are in $H^{1}(0, L)$ and satisfy the corner conditions (2.1). Let $\left(u^{\varepsilon}, v^{\varepsilon}, w^{\varepsilon}\right)$ be solutions of (1.1) (1.2) (1.3).

(i) The $\left(u^{\varepsilon}, v^{\varepsilon}\right)$ are bounded in $L_{t}^{\infty}\left([0, T] ; L_{x}^{2}([0, L])\right)$ and in $L_{t}^{2}\left([0, T] ; L_{x}^{\infty}([0, L])\right)$. Moreover, $\left(\partial_{x} u^{\varepsilon}, \partial_{x} v^{\varepsilon}\right)$ and $\left(\varepsilon \partial_{t} u^{\varepsilon}, \varepsilon \partial_{t} v^{\varepsilon}\right)$ are bounded in $L^{2}([0, T] \times[0, L])$;

(ii) The $w^{\varepsilon}$ are bounded in $L^{\infty}([0, T] \times[0, L]) \cap H^{1}([0, T] \times[0, L])$;

(iii) $\left(u^{\varepsilon}, v^{\varepsilon}, w^{\varepsilon}\right)$ converges in $\left[L^{2}([0, T] \times[0, L])\right]^{3}$ to $\left(u_{*}, v_{*}, w_{*}\right)$ the solution of (1.6) (1.7) (1.8).

The proof has several ingredients:

1) We first prove a preliminary result concerning systems like (4.1). Resolving the boundary conditions, we are led to consider systems of the form

$$
\varepsilon \partial_{t} U+M_{W} U=0, \quad u(0)=v(L)=0,
$$

where $W$ is slowly varying in time. The main result is the exponential decay of the energy of solutions, that is of order $O\left(e^{-\gamma t / \varepsilon}\right)$.

2) Next we apply this result to (4.1) to prove uniform $L^{\infty}$ bounds in time for $U^{\varepsilon}=\left(u^{\varepsilon}, v^{\varepsilon}\right)$. Using the equation (4.3) and the known estimates for $w^{\varepsilon}$, this implies that the family $\left\{w^{\varepsilon}\right\}$ is bounded in $H^{1}([0, T] \times[0, T])$.

3) Therefore, there are subsequences which converge strongly to $w_{*}$ in $L^{2}(\Omega)$. Using the decay of energy once more, we show that $U^{\varepsilon}$ converges strongly to $U_{*}$ which satisfies $\varepsilon \partial_{t} U_{*}+M_{w_{*}} U_{*}=0$, with the right boundary conditions. Hence $\left(U_{*}, w_{*}\right)$ is a solution of the limiting problem. By uniqueness of this solution $\left(U_{*}, w_{*}\right)$, this implies that the full family $\left(U^{\varepsilon}, w^{\varepsilon}\right)$ converges.

\subsection{The fast system; exponential decay of energy.}

For a given potential $W \in C\left([0, T] ; L_{x}^{2}([0, L])\right)$, denote by $\mathcal{E}_{W}^{\varepsilon}(t, s) V$ the value $U(t)$ of the solution at time $t$ (larger than or equal to $s$ ) of the equation

$$
\varepsilon \partial_{t} U+M_{W} U=0,\left.\quad U\right|_{\tau=s}=V
$$


with initial data $V \in\left[L_{x}^{2}\right]^{2}$ and boundary conditions $u(t, 0)=0, \quad v(t, L)=0$. The conservation $\partial_{t}\left(|u|^{2}+|v|^{2}\right)+\varepsilon^{-1} \partial_{x}\left(|u|^{2}-|v|^{2}\right)=0$ and the boundary conditions immediately imply that

$$
\left\|\mathcal{E}_{W}^{\varepsilon}(t, s) V\right\|_{L^{2}([0, L])} \leq\|V\|_{L^{2}([0, L])} .
$$

The linear mapping $\mathcal{E}_{W}^{\varepsilon}(t, s)$ satisfies the group property $\mathcal{E}_{W}^{\varepsilon}\left(t, s^{\prime}\right) \mathcal{E}_{W}^{\varepsilon}\left(s^{\prime}, s\right)=\mathcal{E}_{W}^{\varepsilon}(t, s)$. In particular, if $W$ is a bounded potential independant of time, $\mathcal{E}_{W}^{\varepsilon}(t, 0)$ is a continuous contraction semi-group on $L^{2}([0, L])$.

Theorem 4.2. Given constants $C_{0}$ and $C_{1}$, there are $C$ and $\gamma>0$ such that for all $W$ satisfying

$$
\|W\|_{L^{\infty}([0, T] \times[0, L])} \leq C_{0} \quad\|W\|_{H^{1}([0, T] \times[0, L])} \leq C_{1},
$$

then for all $\varepsilon \in] 0,1]$, all $0 \leq s \leq t \leq T$ :

$$
\left\|\mathcal{E}_{W}^{\varepsilon}(t, s)\right\|_{\mathcal{L}\left(L_{x}^{2} ; L_{x}^{2}\right)} \leq C e^{-\gamma(t-s) / \varepsilon}
$$

To prove the result, it is sufficient to show that there is $\delta<1$, such that

$$
\left\|\mathcal{E}_{W}(s+2 \varepsilon L, s)\right\|_{\mathcal{L}\left(L^{2} ; L^{2}\right)} \leq \delta
$$

for all $s \in[0, T-2 \varepsilon L]$ and all $W$ in the given bounded subset of $L^{\infty} \cap H^{1}(\Omega)$. To prove this estimate, stretch and change time $t=s+\varepsilon \tau$, so that the function $\widetilde{U}(\tau)=\mathcal{E}_{W}(s+\varepsilon \tau, s) V$, satisfies

$$
\partial_{\tau} \widetilde{U}+M_{a} \widetilde{U}=0, \quad \widetilde{U}_{\mid \tau=0}=V
$$

with unchanged homogeneous boundary conditions and

$$
a(\tau, x)=W(s+\varepsilon \tau, x)
$$

Note that when $W$ satisfies (4.7) then a remains bounded in $L^{\infty} \cap H^{1}\left(\Omega_{0}\right)$ where $\Omega_{0}=[0,2 L] \times[0, L]$. Therefore, the Theorem follows from the next lemma where one takes $s=0$.

Lemma 4.3. Given a bounded subset in $L^{\infty} \cap H^{1}\left(\Omega_{0}\right)$, there is $\delta<1$ such that for all a in this bounded set, the solution $\widetilde{U}$ of (4.9) satisfies

$$
\|\widetilde{U}(2 L)\|_{L^{2}([0, L])} \leq \delta\|\widetilde{U}(0)\|_{L^{2}([0, L])} .
$$

Proof. The conservation $\partial_{\tau}\left(|\widetilde{u}|^{2}+|\widetilde{v}|^{2}\right)+\partial_{x}\left(|\widetilde{u}|^{2}-|\widetilde{v}|^{2}\right)=0$ implies that

$$
\|\widetilde{U}(T)\|_{L^{2}([0, L])}^{2}=\left\|\widetilde{U}_{\mid \tau=0}\right\|_{L^{2}([0, L])}^{2}-\|\widetilde{u}(\cdot, L)\|_{L^{2}([0,2 L])}^{2}-\|\widetilde{v}(\cdot, 0)\|_{L^{2}([0,2 L])}^{2} .
$$

Hence, it is sufficient to prove that there is $C$ such that for all $a$ in the given bounded susbset of $L^{\infty} \cap H^{1}\left(\Omega_{0}\right)$, the solution $\widetilde{U}$ of (4.9) satisfies:

$$
\left\|\widetilde{U}_{\mid \tau=0}(\cdot)\right\|_{L^{2}([0, L])}^{2} \leq C\left(\|\widetilde{u}(\cdot, L)\|_{L^{2}([0,2 L])}^{2}+\|\widetilde{v}(\cdot, 0)\|_{L^{2}([0,2 L])}^{2}\right) .
$$

If not, there are sequences $a^{n}, U^{n}$ such that

$$
\begin{aligned}
& \left\|U_{\mid t=0}^{n}\right\|_{L^{2}}=1, \\
& \left\|\left.u^{n}(\cdot, L)\right|_{L^{2}([0, T])}+\right\| v^{n}(\cdot, 0) \|_{L^{2}([0, T])} \rightarrow 0 .
\end{aligned}
$$


Extracting a subsequence if necessary, we can assume that $U^{n}$ converges weakly to a limit $U$ in $L^{2}$ and that $a^{n}$ converges weakly to $a$ in $L^{\infty} \cap H^{1}$ and strongly in $H^{\sigma}$ for all $\sigma<1$. In particular, this implies the following strong convergence:

$$
a^{n} \rightarrow a \text { in } L_{t}^{\infty}\left(0,2 L ; L_{x}^{2}([0, L])\right) .
$$

Therefore, $a^{n} v^{n}$ and $\overline{a^{n}} u^{n}$ converge weakly to $a v$ and $\bar{a} u$ respectively. Hence, we can pass to the weak limit in the equations

$$
\left\{\begin{array}{l}
\partial_{\tau} u^{n}+\partial_{x} u^{n}=-a^{n} v^{n} \\
\partial_{\tau} v^{n}-\partial_{x} v^{n}=\overline{a^{n}} u^{n}
\end{array}\right.
$$

to find that $U$ satisfies

$$
\left\{\begin{array}{l}
\partial_{\tau} u+\partial_{x} u=-a v \\
\partial_{\tau} v-\partial_{x} v=\bar{a} u
\end{array}\right.
$$

The right hand sides in (4.13) are bounded in $L^{2}$, implying that the traces on the boundary are well defined and moreover that $U^{n}{ }_{\mid x=0} \rightarrow U_{\mid x=0}$ weakly, with similar results for the traces on $x=L$ and $t=0$. With (4.11) this implies the strong convergence of the traces on the lateral boundaries:

$$
U_{\mid x=0}^{n} \rightarrow U_{\mid x=0}=0 \quad \text { and } \quad U_{\mid x=L}^{n} \rightarrow U_{\mid x=L}=0 \quad \text { in } L^{2}([0,2 L]) .
$$

Thus, $U$ and $U^{n}$ are weak=strong solutions of (4.9) with potential $a$ and $a^{n}$ respectively. This implies the conservation law

$$
\left(\partial_{\tau}-\partial_{x}\right)\left|v^{n}-v\right|^{2}=f^{n}:=2 \operatorname{Re} \bar{a}\left(u^{n}-u\right)\left(\bar{v}^{n}-\bar{v}\right)+\left(\bar{a}^{n}-\bar{a}\right) u^{n}\left(\bar{v}^{n}-\bar{v}\right) .
$$

Integrating over the the triangle $\Delta:=\{0 \leq t, 0 \leq x, t+x \leq L\}$ yields:

$$
\left\|\left(v^{n}-v\right)_{\mid t=0}\right\|_{L^{2}([0, L])}^{2}=\left\|\left(v^{n}-v\right)_{\mid x=0}\right\|_{L^{2}([0, L])}^{2}-\int_{\Delta} f^{n} d t d x .
$$

The classical energy estimates for (4.9) imply that $U^{n}, U$ and thus $U^{n}-U$ are bounded in $L_{t}^{\infty}\left(L_{x}^{2}\right)$. By Proposition 2.6 they are also bounded in $L_{t}^{2}\left(L_{x}^{\infty}\right)$. Thus $u^{n}\left(\bar{v}^{n}-\bar{v}\right)$ is bounded in $L_{t}^{1}\left(L_{x}^{\infty}\right)$ and by (4.12), this implies that

$$
\left(\bar{a}^{n}-\bar{a}\right) u^{n}\left(\bar{v}^{n}-\bar{v}\right) \rightarrow 0 \quad \text { in } L^{1}(\Omega) .
$$

Moreover, $\left(u^{n}-u\right)\left(\bar{v}^{n}-\bar{v}\right)$ is bounded in $L_{t x}^{1}$. Since $\left(\partial_{t}+\partial_{x}\right)\left(u^{n}-u\right)$ and $\left(\partial_{t}-\partial_{x}\right)\left(v^{n}-v\right)$ are bounded in $L_{t, x}^{2}$ by basic compensated compactness, the product $\left(u^{n}-u\right)\left(\bar{v}^{n}-\bar{v}\right)$ converges in the sense of distributions to 0 . This implies that

$$
\int_{\Delta} \bar{a}\left(u^{n}-u\right)\left(\bar{v}^{n}-\bar{v}\right) d t d x \rightarrow 0, \quad \int_{\Delta} f^{n} d t d x \rightarrow 0 .
$$

With (4.15) and (4.16), this implies that $v^{n}{ }_{\mid t=0}$ converges strongly in $L^{2}$. Similarly, $u_{\mid t=0}^{n}$ converges strongly in $L^{2}$. Hence, by (4.10)

$$
\left\|U_{\mid t=0}\right\|_{L^{2}}=1 .
$$

Therefore the contradiction which implies Lemma 4.3 and Theorem 4.2 is a consequence of the next Lemma 4.4. 
Lemma 4.4. Suppose that $W \in L^{\infty}([0, T] \times[0, L])$ and $U \in L^{2}([0, T] \times[0, L])$ satisfies $\partial_{\tau} U+M_{W} U=0$, $U_{\mid x=0}=0$ and $U_{\mid x=L}=0$. If $T>L$, then $U=0$.

Proof. By hyperbolicity in the $x$ direction, and local uniqueness of the Cauchy problem with data on $x=0, U=0$ on the triangle $\Delta_{1}=\{0 \leq x \leq L, x \leq t \leq T-x\}$. Similarly, $U=0$ on the triangle $\Delta_{2}=\{0 \leq x \leq L, L-x \leq t \leq T-L+x\}$. Because $T>L$, the two triangles intersect, and their union contain a neighborhood of the line $t=T / 2$. Therefore, by uniqueness of the Cauchy problem in time for the boundary value problem (4.5), $U=0$.

\subsection{The initial layer and uniform $L^{\infty}$ bounds for $u$ and $v$.}

Theorem 4.5. Suppose that the initial data $u_{0}, w_{0}$ are in $H^{1}(0, L)$ and satisfy the corner conditions (2.1). Let $\left(u^{\varepsilon}, v^{\varepsilon}, w^{\varepsilon}\right)$ be solutions of (1.1) (1.2) (1.3). Then $u^{\varepsilon}$ and $v^{\varepsilon}$ are bounded in $L^{\infty}\left(0, T ; L^{2}([0, L])\right)$ for $\left.\left.\varepsilon \in\right] 0, \frac{1}{2}\right]$.

Proof. We study the fast system (4.1) considering $w^{\varepsilon}$ as known and using the estimates proved in Sections 2 and 3. The idea is that after a small initial layer, $U^{\varepsilon}(t)$ is close to $\underline{U}^{\varepsilon}(t)=u^{i n}(t) \mathcal{Y}\left(w^{\varepsilon}(t)\right)$ which, for all $t$, is the stationary solution of

$$
M_{w^{\varepsilon}(t)} \underline{U}^{\varepsilon}(t)=0
$$

with the boundary conditions

$$
\underline{u}(t, 0)=u^{i n}(t), \quad \underline{v}(t, L)=0 .
$$

According to Proposition 3.1, $\left\|\underline{U}^{\varepsilon}(t)\right\|_{L_{x}^{2}}$ is uniformely bounded.

Denote now $\widetilde{U}$ the solution of

$$
\varepsilon \partial_{t} \widetilde{U}+M_{w_{0}} \widetilde{U}=0
$$

with homogeneous boundary conditions $\tilde{u}^{\varepsilon}(t, 0)=\tilde{v}^{\varepsilon}(t, L)=0$ and initial conditions

$$
\widetilde{U}^{\varepsilon}(0)=-u^{i n} \mathcal{Y}\left(w_{0}\right)=(-\underline{u}(0, .),-\underline{v}(0, .))
$$

( $\widetilde{U}$ is like an initial layer).According to Theorem 4.2 we get

$$
\|\widetilde{U}(t)\|_{L_{x}^{2}}=\left\|u^{i n}\right\|_{L^{\infty}}\left\|\mathcal{E}_{w_{0}}^{\varepsilon}(t, 0) \mathcal{Y}\left(w_{0}\right)\right\|_{L_{x}^{2}} \leq C e^{-\gamma t / \varepsilon} .
$$

Therefore, to prove the theorem, it is sufficient to show that $U^{\sharp}=U^{\varepsilon}-\underline{U}^{\varepsilon}-\widetilde{U}$ is bounded in $\left.L^{\infty}\left(0, T ; L_{x}^{2}\right)\right) . U^{\sharp}$ satisfies

$$
\varepsilon \partial_{t} U^{\sharp}+M_{w^{\varepsilon}} U^{\sharp}=F^{\varepsilon},
$$

with homogneneous boundary conditions $u^{\sharp}(t, 0)=v^{\sharp}(t, L)=0$ and initial conditions $u^{\sharp}(0, x)=$ $u_{0}(x), v^{\sharp}(0, x)=0$, and

$$
F^{\varepsilon}=-\varepsilon \partial_{t} \underline{U}^{\varepsilon}-\left(w^{\varepsilon}-w_{0}\right) \perp \widetilde{U}^{\varepsilon}=\left(\begin{array}{c}
-\varepsilon \partial_{t} \underline{u^{\varepsilon}}-\left(w^{\varepsilon}-w_{0}\right) \widetilde{v} \\
-\varepsilon \partial_{t} \underline{v^{\varepsilon}}+\left(\overline{w^{\varepsilon}}-\bar{w}_{0}\right) \widetilde{u}
\end{array}\right) .
$$


Classical energy estimates for solutions of (4.22) with homogeneous boundary conditions, imply that for all $t_{0} \leq T$

$$
\left\|U^{\sharp}\left(t_{0}\right)\right\|_{L_{x}^{2}} \leq\left\|u_{0}\right\|_{L_{x}^{2}}+\frac{1}{\varepsilon} \int_{0}^{t_{0}}\left\|F^{\varepsilon}(t)\right\|_{L_{x}^{2}} d t
$$

Therefore, it remains to show that there is $C$ such that for all $\left.\varepsilon \in] 0, \frac{1}{2}\right]$

$$
\int_{0}^{T}\left\|F^{\varepsilon}(t)\right\|_{L_{x}^{2}} d t \leq C \varepsilon
$$

The first term in $F^{\varepsilon}$ is

$$
\varepsilon \partial_{t} \mathcal{Y}\left(w^{\varepsilon}(t)\right)=\varepsilon \lim _{h} \frac{1}{h}\left[u^{i n}(t+h) \mathcal{Y}\left(w^{\varepsilon}(t+h)\right)-u^{i n}(t) \mathcal{Y}\left(w^{\varepsilon}(t)\right)\right] .
$$

By Theorem 2.1, the $w^{\varepsilon}$ are uniformly bounded in $L^{\infty}$ and therefore Proposition 3.1 implies that for some constant $C$ independent of $\varepsilon$ :

$$
\varepsilon\left\|\partial_{t} \underline{U}^{\varepsilon}(t)\right\|_{L_{x}^{2}} \leq \varepsilon C\left(\left\|\partial_{t} w^{\varepsilon}(t)\right\|_{L_{x}^{2}}+\left|\partial_{t} u^{i n}(t)\right|\right) .
$$

The second term in $F^{\varepsilon}$ is $\left(w^{\varepsilon}-w_{0}\right) \perp \widetilde{U}$. By $(4.21)$ and the $L^{\infty}$ bounds for $w^{\varepsilon}$, it satisfies

$$
\left.\|\left(w^{\varepsilon}-w_{0}\right) \perp \tilde{U} t\right) \|_{L^{2}} \leq C e^{-\gamma t / \varepsilon} .
$$

Integrating these two estimates over $[0, T]$ imply (4.24) and the theorem follows.

Corollary 4.6. Under the assumptions of the theorem, $u^{\varepsilon} v^{\bar{\varepsilon}}$ belongs to a bounded set in $L^{2}([0, T] \times$ $[0, L])$. Moreover $\partial_{t} w^{\varepsilon}$ is bounded in $L^{2}([0, T] \times[0, L])$ and $w^{\varepsilon}$ is bounded in $H^{1}([0, T] \times[0, L])$.

Proof. By Proposition 2.6, $u^{\varepsilon}$ is bounded in $L_{t}^{2}\left(L_{x}^{\infty}\right)$. The theorem asserts that $v^{\varepsilon}$ is bounded in $L_{t}^{\infty}\left(L_{x}^{2}\right)$. Thus $u^{\varepsilon} \bar{v}^{\varepsilon}$ is bounded in $L^{2}([0, T] \times[0, L])$. $L^{2}(\Omega)$.

By Theorem 2.7, $\partial_{x} w^{\varepsilon}$ is bounded in $L^{2}(\Omega)$ and therefore $\partial_{t} w^{\varepsilon}=-\partial_{x} w^{\varepsilon}+u^{\varepsilon} \bar{v}^{\varepsilon}$ is bounded in

\subsection{Strong convergence. End of proof of Theorem 4.1}

Therefore the products $u^{\varepsilon} \overline{v^{\varepsilon}}, v^{\varepsilon} w^{\varepsilon}$ and $u^{\varepsilon} \overline{w^{\varepsilon}}$ are uniformely bounded in $L^{2}(\Omega)$. To finish the proof of Theroem 4.1 it suffices to prove the convergence of a subsequence of the solutions $\left(u^{\varepsilon}, v^{\varepsilon}, w^{\varepsilon}\right)$ of $(4.1)(4.2)(4.3)$ :

$$
\left(u^{\varepsilon}, v^{\varepsilon}, w^{\varepsilon}\right) \rightarrow\left(u_{*}, v_{*}, w_{*}\right), \quad \text { in }\left[L^{2}([0, T] \times[0, L])\right]^{3} .
$$

Indeed, knowing this strong convergence, we can pass to the limit in the quadratic terms in the equations and therefore $\left(u_{*}, v_{*}, w_{*}\right)$ is a solution of $(1.6)(1.7)(1.8)$. By uniqueness of the solution of the limit problem, this implies the convergence of the full family.

Proof of (4.25). The $w^{\varepsilon}$ are bounded in $L^{\infty}([0, T] \times[0, L]) \cap H^{1}([0, T] \times[0, L])$, thus there is a subsequence which converges to a function $w_{*}$ strongly in $C\left([0, T], L^{2}([0, L])\right)$. In particular $w_{* \mid t=0}=$ $w_{0}$. Denote $U_{*}(t)$ the vector function which solves for all t the system

$$
M_{w_{*}(t)} U_{*}=0, \quad u_{*}(0)=u^{i n}, \quad v_{*}(L)=0 .
$$


The proof of the strong convergence of $U^{\varepsilon}$ is parallel to the proof of Theorem 4.5. We use the same vector function $\underline{U}^{\varepsilon}(t)=u^{i n}(t) \mathcal{Y}\left(w^{\varepsilon}(t)\right)$ as above, it satisfies:

$$
M_{w^{\varepsilon}(t)} \underline{U}^{\varepsilon}=0 .
$$

According to Proposition 3.1, there exists $C$ such that

$$
\left\|\mathcal{Y}\left(w^{\varepsilon}(t)\right)-\mathcal{Y}\left(w_{*}(t)\right)\right\|_{\left[L_{x}^{2}\right]^{2}} \leq C\left\|w^{\varepsilon}(t)-w_{*}(t)\right\|_{L_{x}^{2}} \quad \forall t \leq T,
$$

and hence $\underline{U}^{\varepsilon}(t)$ converges strongly to $U_{*}$ in $L_{t, x}^{2}$.

To describe the initial layer, introduce the solution $\widetilde{U}^{\varepsilon}=\mathcal{E}_{w^{\varepsilon}}^{\varepsilon}(t, 0)\left(U_{\mid t=0}^{\varepsilon}-\underline{U}_{\mid t=0}^{\varepsilon}\right)$ of

$$
\varepsilon \partial_{t} \widetilde{U}^{\varepsilon}+M_{w^{\varepsilon}} \widetilde{U}^{\varepsilon}=0, \quad \widetilde{U}_{\mid t=0}^{\varepsilon}=U_{\mid t=0}^{\varepsilon}-\underline{U}_{\mid t=0}^{\varepsilon}, \quad \tilde{u}(t, 0)=\tilde{v}(L, t)=0 .
$$

Theorem 4.2 and Corollary 4.6 imply that there exist $C$ and $\gamma>0$ such that for all $\varepsilon$ and all $t \in[0, T]$ :

$$
\left\|\widetilde{U}^{\varepsilon}(t)\right\|_{\left[L_{x}^{2}\right]^{2}} \leq C e^{-\gamma t / \varepsilon}
$$

Let $U_{\sharp}^{\varepsilon}=U^{\varepsilon}-\underline{U}^{\varepsilon}-\widetilde{U}^{\varepsilon}$. It satisfies

$$
\varepsilon \partial_{t} U_{\sharp}^{\varepsilon}+M_{w^{\varepsilon}} U_{\sharp}^{\varepsilon}=-\varepsilon \partial_{t} \underline{U}^{\varepsilon}
$$

with homogneneous boundary and initial conditions. Therefore, by Duhamel's principle,

$$
U_{\sharp}^{\varepsilon}(t)=-\int_{0}^{t} \mathcal{E}_{W}(t, s)\left(\partial_{t} \underline{U}^{\varepsilon}\right)(s) d s=-\int_{0}^{t} \mathcal{E}_{W}(t, s) \partial_{t}\left[u^{i n} \mathcal{Y}\left(w^{\varepsilon}(s)\right)\right] d s
$$

Since $\partial_{t} \mathcal{Y}\left(w^{\varepsilon}(t)\right)=\lim _{h \rightarrow 0} \frac{1}{h}\left[\mathcal{Y}\left(w^{\varepsilon}(t+h)\right)-\mathcal{Y}\left(w^{\varepsilon}(t)\right)\right]$, according to uniform bound of $\left\|w^{\varepsilon}\right\|_{L_{t, x}^{\infty}}$ and to Proposition 3.1, we have

$$
\left\|\partial_{t} \mathcal{Y}\left(w^{\varepsilon}(t)\right)\right\|_{L_{x}^{2}} \leq C\left(w_{\infty}\right)\left\|\partial_{t} w^{\varepsilon}(t)\right\|_{L_{x}^{2}}
$$

By corollary 4.6, one knows that $\left\|\partial_{t} w^{\varepsilon}(t)\right\|_{L_{x}^{2}}$ is in $L^{2}(0, T)$. Thus, thanks to theorem 4.2, there exists a positive constant $\gamma$ such that

$$
\begin{aligned}
\left\|U_{\sharp}^{\varepsilon}(t)\right\|_{L_{x}^{2}} & \leq C \int_{0}^{t} e^{-\gamma(t-s) / \varepsilon}\left(\left\|\partial_{t} w^{\varepsilon}(s)\right\|_{L_{x}^{2}}+\left|\partial_{t} u^{i n}(s)\right|\right) d s \\
& \leq C\left(\int_{0}^{t} e^{-2 \gamma s / \varepsilon} d s\right)^{1 / 2}\left(\left\|\partial_{t} w^{\varepsilon}\right\|_{L_{t, x}^{2}}+\left\|\partial_{t} u^{i n}\right\|_{L_{t}^{2}}\right)=\sqrt{\varepsilon} C\left(\left\|\partial_{t} w^{\varepsilon}\right\|_{L_{t, x}^{2}}+C^{\prime}\right) .
\end{aligned}
$$

Therefore, $U^{\varepsilon}(t)$ converges strongly to $U_{*}(t)=u^{i n}(t) \mathcal{Y}\left(w_{*}\right)$ in $L_{x}^{2}$ for all $t$ and (4.25) holds. The proof of the main theorem is now complete. 


\section{Appendix. A physical glance on the three-wave coupling for Brillouin instability.}

Several physical phenomena may occur when high power laser beams propagates in hot plasmas. Different typical lengths may be found which are related to these different phenomena: the typical length $L_{p l}$ of variation of the mean density of the plasma; the typical length $L_{l}$ of variation of the amplitude of the laser intensity, $2 \pi L_{l}$ is in the order of the width of the speckles (which are hot spots of light in the laser beam) and of course the laser wave length in the vacuum $2 \pi / k_{0}$.

Generally one has $2 \pi / k_{0} \ll 2 \pi L_{l} \ll L_{p l}$; for example, for the high power intensity laser beam, the wave length is equal to a fraction of one micron, $L_{l}$ is typically of order of one micron (then $k_{0} L_{l} \approx 10$ ) and $L_{p l}$ is larger than 100 microns.

Beside the classical diffraction and the absorption by the plasma, since the refraction index of the plasma depends on its density, there is refraction of light at the macroscopic level (characterized by $L_{p l}$ ) and at the scale of the width of the speckles (characterized by $L_{l}$ ) which produces a self-focusing of these speckles. But here we address only the coupling between the main laser wave and an ion acoustic wave which creates the so called stimulated Brillouin backscattered laser wave. Moreover in these plasmas, the ion acoustic waves propagates with a speed which is in the order of $10^{-3}$ of the speed of light.

The derivation of the coupling model has been performed for a long time but it is quite tricky; for a recent physical introduction to this three-wave coupling modeling, see for example [2],[13],[19] (see also [20] for an mathematical introduction to this derivation). We only give the outlines of this derivation. We briefly derive a first coupling model (see (5.4)(5.5)(5.6) ) and afterwards we focus on simplifications of this system in order to obtain the so-called standard decay model which is the three-wave coupling system we address in this paper.

\section{First coupling model.}

Denote by $c$, the speed of light. The laser pulsation is $\omega_{0}=c k_{0}$. It is classical to use the dimensionless electron density normalized with the critical density (defined by $q_{e}^{-2} \omega_{0}^{2} \varepsilon_{0} m_{e}$ where $q_{e}, m_{e}$ denote the electron charge and mass and $\varepsilon_{0}$ the vacuum permeability). One assumes here that the electron plasma density it quite constant, i.e.

$$
N(t, \mathbf{x})=N_{\text {ref }}(1+n(t, \mathbf{x})), \quad n \ll 1,
$$

where $N_{\text {ref }}$ is the mean density which is stricly smaller than 1 (and in pratice less than 0.5 ) and $n$ is the density perturbation. The laser wave is represented by the electromagnetic field $\Psi=\Psi(t, \mathbf{x})$ satisfying the following wave equation (derived from the full Maxwell ones)

$$
\frac{\partial^{2}}{\partial t^{2}} \Psi-c^{2} \Delta \Psi+\omega_{p}^{2}(1+n) \Psi=0 .
$$

where the plasma frequency $\omega_{p}$ is given by

$$
\omega_{p}^{2}=\omega_{0}^{2} N_{\text {ref }}
$$

The laser beam is assumed to travel in the fixed direction characterized by the unit vector $\mathbf{e}_{z}$, denote $z=\mathbf{x} . \mathbf{e}_{z}$. One introduces $E=E(t, \mathbf{x})$ the space-time envelope of the field corresponding to the main wave traveling in the direction $\mathbf{e}_{z}$ and $\Phi(t, \mathbf{x})$ the one corresponding to the backscattered wave; they are complex functions and are slowly varying with respect to the time and space variables $\mathbf{x}$

$$
\Psi(t, \mathbf{x})=\left[E(t, \mathbf{x}) e^{i k_{p} z-i \omega_{0} t}+c . c\right]+\left[\Phi(t, \mathbf{x}) e^{-i k_{p} z-i \omega_{0} t}+c . c .\right]
$$


where $k_{p}$ solves the classical dispersion relation

$$
k_{p}=\frac{1}{c} \sqrt{\omega_{0}^{2}-\omega_{p}^{2}}=k_{0} \sqrt{1-N_{\text {ref }}}
$$

Denote $c_{g}=c \sqrt{1-N_{\text {ref }}}$ the so-called group velocity and $\beta_{0}=\frac{\omega_{p}^{2}}{2 \omega_{0}}$. The two waves satisfiy the paraxial equations obtained by assuming that $k_{p}^{-1}$ and $\omega_{0}^{-1}$ are small enough compared to the characteristic values of the space and time variation of $E$ and $\Phi$

$$
\begin{aligned}
\frac{\partial}{\partial t} E+c_{g} \partial_{z} E-\frac{i c}{2 k_{0}} \Delta_{\perp} E & =-i \beta_{0} n e^{-2 i k_{p} z} \Phi \\
\frac{\partial}{\partial t} \Phi-c_{g} \partial_{z} \Phi-\frac{i c}{2 k_{0}} \Delta_{\perp} \Phi & =-i \beta_{0} n e^{2 i k_{p} z} E
\end{aligned}
$$

where the diffraction operators $i \Delta_{\perp}$ correspond to a diffusion in the directions transverse to $\mathbf{e}_{z}$. In the sequel we does not account for these transverse effects. Notice that the quantity $n$ is highly oscillating with respect to the space variable (see below) thus terms like $\beta_{0} n E$ and $\beta_{0} n \Phi$ which appear theoreticaly in the expansion of the first and second equation respectively are also highly oscillating with respect to the space and so they have to be withdrawn. The laser field produces a ponderomotive force in the plasma which is proportional to $\nabla|\Psi|^{2}$, so neglecting the transverse phenomena, it reduces to a term proportional to $2 i k_{p} E \bar{\Phi} e^{2 i k_{p} z}$; it generates a wave, called ion acoustic wave corresponding to the wave number $k_{s}=2 k_{p}$. Therefore for plasma response, the simplest model is the following ( $q c_{s}$ corresponds to the velocity of the ion acoustic wave)

$$
\begin{aligned}
\frac{\partial}{\partial t} n+c_{s} \partial_{z} q & =0 \\
\frac{\partial}{\partial t} q+c_{s} \partial_{z} n+2 \nu_{L} q & =-\gamma_{p} c_{s}^{-1}\left(i k_{s} E \bar{\Phi} e^{i k_{s} z}+\text { c.c. }\right)
\end{aligned}
$$

the term of the form $\nu_{L} q$ is related to the Landau damping effect and $\gamma_{p}$ is a constant depending only of the characteristics of the plasma ions. We may now take the space envelope $M$ (which is not highly oscillating with respect to the space variable) of $n$, i.e. we set

$$
n(t, z)=M(t, z) e^{i k_{s} z}+c . c .
$$

thus, neglecting as above the terms $\partial_{z} \Phi, \partial_{z} E$ with respect to $i k_{s} \Phi, i k_{s} E$, the previous system is equivalent to the following equation for $M$

$$
\frac{\partial^{2}}{\partial t^{2}} M-c_{s}^{2}\left(\partial_{z}+i k_{s}\right)^{2} M+2 \nu_{L} \frac{\partial}{\partial t} M=-\gamma_{p} k_{s}^{2} E \bar{\Phi}
$$

After withdrawing the terms $\bar{M} \Phi e^{-2 i k_{s} z}$ and $M E e^{2 i k_{s} z}$ which are highly oscillating with respect to the space variable, the propagation equations for $E$ and for $\Phi$ may read as

$$
\begin{aligned}
\frac{\partial}{\partial t} E+c_{g} \partial_{z} E & =-i \beta_{0} M \Phi \\
\frac{\partial}{\partial t} \Phi-c_{g} \partial_{z} \Phi & =-i \beta_{0} \bar{M} E
\end{aligned}
$$


Equations (5.4)(5.5)(5.6) make up the so-called modified decay model for the Brillouin instability. Of course, it has to be supplemented by initial conditions $E(0,),. \Phi(0,),. M(0,$.$) and boundary condi-$ tions on both sides of the simulation interval for $M$ and on the boundaries $z=0$ for $E$ and $z=L$ for $\Phi$.

\section{The standard decay model.}

The derivation of this model is based on a linearization of the previous one. So we assume for a while that the field $E$ is constant, the system reduces to $(5.2)(5.3)(5.6)$. We address this one from stability analysis point of view assuming it is posed on the whole space $\mathbf{R}$. In the case when $\nu_{L}$ is set to zero, after introducing the space envelopes $m$ and $s$ of the two traveling waves $(n+q) / 2$ and $(n-q) / 2$

$$
\frac{n+q}{2}=m(z) e^{i k_{s} z}+c . c ., \quad \frac{n-q}{2}=s(z) e^{i k_{s} z}+c . c .,
$$

we get the following advection equations for $m, s$ and $\Phi$ (in this last one, the highly oscillating term $(m+s) E e^{2 i k_{s} z}$ has to be withdrawn)

$$
\begin{aligned}
\frac{\partial}{\partial t} m+c_{s}\left(i k_{s}+\partial_{z}\right) m & =-i \frac{\gamma_{p} k_{s}}{2 c_{s}} E \bar{\Phi} \\
\frac{\partial}{\partial t} s-c_{s}\left(i k_{s}+\partial_{z}\right) s & =i \frac{\gamma_{p} k_{s}}{2 c_{s}} E \bar{\Phi} \\
\left(\frac{\partial}{\partial t}-c_{g} \partial_{z}\right) \Phi & =-i \beta_{0}(\bar{m}+\bar{s}) E
\end{aligned}
$$

First, disregarding the $s$-wave, we search $m^{\sharp}$ and $\phi^{\sharp}$ constants such that $m(t, z)=m^{\sharp} e^{-i \Omega t} e^{i \zeta z}$ and $\Phi(t, z)=E \phi^{\sharp} e^{i \Omega t} e^{-i \zeta z}$. So the corresponding dispersion relation is

$$
\left|\begin{array}{cc}
\Omega-c_{s}\left(\zeta+k_{s}\right) & -\frac{1}{2} \gamma_{p} k_{s} c_{s}^{-1}|E|^{2} \\
\beta_{0} & \Omega+c_{g} \zeta
\end{array}\right|=0
$$

Then, denoting $\varepsilon=c_{s} / c_{g}$, the ratio between the sound speed and the light speed and $B=|E| \varepsilon^{-1 / 2} k_{0}$ $\sqrt{N_{\text {ref }} \gamma_{p} / 2}$, the discriminant of this second order equation is equal to $c_{s}^{2}\left(k_{s}+\frac{1}{\varepsilon} \zeta(1+\varepsilon)\right)^{2}-4 B^{2}$. The growth of the instability corresponds to the case where there exists a couple $\left(\Omega_{\zeta}, \zeta\right)$ such that $\operatorname{Im}\left(\Omega_{\zeta}\right)>0$. Since we have $\operatorname{Im}\left(\Omega_{\zeta}\right)=\frac{1}{2} \sqrt{-c_{s}^{2}\left(k_{s}+\frac{1+\varepsilon}{\varepsilon} \zeta\right)^{2}+4 B^{2}}$, the maximum value of this quantity is obtained for the wave number $\zeta_{*}=-k_{s} \frac{\varepsilon}{1+\varepsilon}$ and the instability grows like $\exp \left(t \operatorname{Im}\left(\Omega_{\zeta_{*}}\right)\right)=\exp (t B)$.

For the coupling between $s$ and $\Phi$, one may check that the solution $\Omega$ of the dispersion relation

$$
\left|\begin{array}{cc}
\Omega+c_{s}\left(\zeta+k_{s}\right) & -\frac{1}{2} \gamma_{p} k_{s} c_{s}^{-1}|E|^{2} \\
\beta_{0} & \Omega+c_{g} \zeta
\end{array}\right|=0
$$

is always real for the wave number $\zeta=\zeta_{*}$. Therefore, the corresponding wave $s$ remains bounded when the time increases and we may neglect $s$ with respect to $m$. So in system (5.7)(5.8)(5.9), it is possible to withdraw equation (5.8) and the $s$ term. For the density fluctuation, we get the following approximation

$$
n(t, z)=e^{i k_{s} z} m(t, z)+c . c .
$$

We now go back to the general case where $E$ is not constant; then one supplements (5.7)(5.9) with the evolution equation for the wave $E$ and (after re-introducing the coefficient $\nu_{L}$ ) we get the so-called standard decay model 


$$
\begin{aligned}
\partial_{t} E+c_{g} \partial_{z} E & =-i \beta_{0} m \Phi \\
\partial_{t} \Phi-c_{g} \partial_{z} \Phi & =-i \beta_{0} \bar{m} E \\
\partial_{t} m+\partial_{z} m+\left(\nu_{L}+i c_{s} k_{s}\right) m & =-i \frac{\gamma_{p} k_{s}}{2 c_{s}} E \bar{\Phi}
\end{aligned}
$$

The main mathematical difficulties of the three-wave coupling phenomena may be seen on this system posed on the interval $\left[0, z_{\max }\right]$ and supplemented with the following natural boundary conditions

$$
E(t, 0)=\alpha^{i n}, \quad \Phi\left(t, z_{\max }\right)=0, \quad m(t, 0)=0 .
$$

To get a non trivial solution for (5.10), it sufficient to have $m(0,$.$) equal to a small random noise$ (or to address a boundary condition on $m$ by setting $m(t, 0)$ equal to a small random noise).

\section{Dimensionless form of the system}

Denoting $\alpha_{\text {ref }}$ a charactersitic value of $\alpha^{i n}$, if we define $\widehat{E}, \widehat{\Phi}, \widehat{m}$ by

$$
E=\widehat{E} \alpha_{\mathrm{ref}}, \quad \Phi=\widehat{\Phi} \alpha_{\mathrm{ref}}, \quad m=-i \widehat{m} \frac{\alpha_{\mathrm{ref}}}{c_{s}} \sqrt{2 \gamma_{p} \frac{1-N_{\mathrm{ref}}}{N_{\mathrm{ref}}}} .
$$

then, denoting $\gamma_{0}=\sqrt{N_{\text {ref }} \gamma_{p} / 2} \alpha_{\text {ref }} k_{0} / c_{s}$, the previous system reads

$$
\begin{aligned}
\partial_{t} \widehat{E}+\frac{c_{s}}{\varepsilon} \partial_{z} \widehat{E} & =-\frac{c_{s}}{\varepsilon} \gamma_{0} \widehat{m} \widehat{\Phi}, \\
\partial_{t} \widehat{\Phi}-\frac{c_{s}}{\varepsilon} \partial_{z} \widehat{\Phi} & =\frac{c_{s}}{\varepsilon} \gamma_{0} \widehat{\widehat{m}} \widehat{E_{p}}, \\
\partial_{t} \widehat{m}+\partial_{z} \widehat{m}+\left(\nu_{L}+i c_{s} k_{s}\right) \widehat{m} & =c_{s} \gamma_{0} \widehat{E_{p}} \widehat{\widehat{\Phi}} .
\end{aligned}
$$

So, for the previous problem, there is a characteristic length which is given by $\gamma_{0}{ }^{-1}$ and a characteristic time given by $\left(c_{s} \gamma_{0}\right)^{-1}$. Give now some typical physical values of the problem we are interested in: for a plasma such that $N_{\text {ref }}=0.15$ with a temperature equal to $3.10^{7} \mathrm{~K}$. In a very hot speckle of the laser beam, we may assume that the laser intensity is about $10^{16} \mathrm{~W} / \mathrm{cm}^{2}$, then the corresponding value of the incoming laser field may be set to $\alpha_{\text {ref }}=10^{11} \mathrm{~V} / \mathrm{m}$; moreover we get $c_{g}=2.810^{8}$ and $c_{s}=5.610^{5}$ thus $\varepsilon=.002 ; \omega_{0}=5.10^{15} \mathrm{~s}^{-1}$, so $k_{0}=1.710^{7} \mathrm{~m}^{-1}, \sqrt{\gamma_{p}}=3.10^{-6}$ in S.I. units, thus we get

$$
\gamma_{0}^{-1} \simeq 4 . \quad 10^{-7} \mathrm{~m}, \quad\left(c_{s} \gamma_{0}\right)^{-1} \simeq 0.7 \quad 10^{-12} \mathrm{~s} .
$$

Notice that with these values, the growth coefficient $B$ of the germ of the instability of $(m, \Phi)$ corresponding to $|E|=\alpha_{\text {ref }}$ is such that $B=c_{s} \gamma_{0} \varepsilon^{-1 / 2}$, so it is 20 times larger than $\left(c_{s} \gamma_{0}\right)$. This is another justification from a physical point of view of the replacement of the modified decay model by the above standard decay model.

Let us go back now to the definition of the dimensionless time and space variables

$$
t^{\prime}=c_{s} \gamma_{0} t, \quad x=z \gamma_{0}, \quad \eta=\nu_{L} /\left(c_{s} \gamma_{0}\right) .
$$

So let us perform the change of notations : 


$$
\widehat{E}(t, z)=E_{p}\left(t^{\prime}, x\right), \quad \widehat{\Phi}(t, z)=E_{m}\left(t^{\prime}, x\right), \quad \widehat{m}(t, z)=W\left(t^{\prime}, x\right) .
$$

and we get the following dimensionless system :

$$
\begin{aligned}
\left(\varepsilon \partial_{t^{\prime}}+\partial_{x}\right) E_{p} & =-E_{m} W, \\
\left(\varepsilon \partial_{t^{\prime}}-\partial_{x}\right) E_{m} & =E_{p} \bar{W} \\
\partial_{t^{\prime}} W+\partial_{x} W+\left(\eta+i k_{s}\right) W & =E_{p} \overline{E_{m}} .
\end{aligned}
$$

Lastly, writing $t$ instead of $t^{\prime}$, we set

$$
E_{p}(t, x)=e^{-i(t-\varepsilon x) k_{s} / 2} u, \quad E_{m}(t, x)=e^{i(t+\varepsilon x) k_{s} / 2} v, \quad W(t, x)=w(t, x) e^{-i t k_{s}} .
$$

Then the previous system reads as

$$
\begin{aligned}
\left(\varepsilon \partial_{t}+\partial_{x}\right) u & =-w v \\
\left(\varepsilon \partial_{t}-\partial_{x}\right) v & =\bar{w} u \\
\left(\partial_{t}+\partial_{x}\right) w+\eta w & =u \bar{v},
\end{aligned}
$$

This is the Boyd-Kadomstev system we address in this paper in the special case $\eta=0$ (which is the most difficult one since there is no absorption of energy). Of course it is supplemented with boundary conditions and initial conditions like the ones stated in the introduction, in particular with $w_{t=0}$ much smaller than 1.

\section{References}

[1] Ph. Ballereau, M. Casanova, F. Duboc, D. Dureau, H. Jourdren, P.Loiseau, J. Metral, O. Morice, R. Sentis. Coupling Hydrodynamics with a Paraxial Solver for Laser Propagation. J. Sci. Comp. 33, p.1-24 (2007).

[2] R. L. Berger, C. H. Still and E. A. Williams, A. B. Langdon. On the dominant subdominant behavior of stimulated Raman and Brillouin scattering. Phys. of Plasmas, 5, p.4337 (1998).

[3] J. M. Boyd and J. G. Turner, Lagrangian studies of plasma wave interaction. J. Physics A: Gen. Phys., 5, p.881-896, 1972.

[4] M. Colin and T. Colin, On a quasilinear Zakharov system describing laser-plasma interaction, Differential Integral Equations, 17(2004)297-330.

[5] M. Colin and T. Colin, A numerical model for the Raman amplification for laser-plasma interaction, J. Comput. Applied Maths, 193 p.535-562, (2006).

[6] M. Colin, T. Colin, and G. Métivier, Nonlinear models for laser-plasma interactions, Séminaire X-EDP, Ecole Polytéchnique, (2007)X1-X10.

[7] J-L. Joly, G. Métivier, J. Rauch, Transparent Nonlinear Geometric Optics and Maxwell-Bloch Equations. J. of Diff. Equ., 166 (2000) p. 175-250. 
[8] J-L. Joly, G. Métivier, J. Rauch, Coherent and focusing multidimensional nonlinear geometric optics, Ann. Sci. Ecole Norm. Sup. 28, p.51-113, (1995).

[9] B.B. Kadomtsev. Plasma Turbulence. Academic Press, 1965. Translated from the Russian of a book edited in 1964.

[10] S. Klainerman and A. Majda, Singular perturbations of quasilinear hyperbolic systems with large parameters and the incompressible limit of compressible fluids. Comm. Pure Appl. Math. 34, p.481-524 (1981).

[11] E.M. Klein, Singular integrals and differentiability properties. Princeton Univ. Press, Princeton (1970).

[12] D. Pesme, G. Laval, M. Pellat, Parametric instability, Physics Review Let. 31 p. 203 (1973).

[13] P. Loiseau et al. Laser Beam smoothing induced by stimulated Brillouin scatter. Physics Review Letters, 97, p.205001, (2006).

[14] G. Métivier, The Mathematics of Nonlinear Optics Handbook of Differential Equations: Evolutionary Equations Vol. 5, Elsevier (to appear).

[15] G. Métivier and S. Schochet, The incompressible Limit of the Nonisentropic Euler Equations. Arch. Rat. Mech. Anal., 158, p.61-90 (2001).

[16] Ph. Mounaix, D. Pesme, M. Casanova. Nonlinear reflectivity of an inhomogeneous plasma. Phys. Review E, 55, p.4653-4664 (1997).

[17] S. Novikov, V. E. Zakharov et al. Theory of solitons. Consultant Bureau New York, 1984.

[18] D. Pesme. Interaction collisionnelle et collective (chap 2) in La fusion par Confinement Inertiel I. Interaction laser-matière. R. Dautray-Watteau ed., Eyrolles, Paris (1995).

[19] S. Hüller, P.E. Masson-Laborde et al., Harmonic Decomposition to describe the nonlinear evolution of stimulated Brillouin Scattering Phys. of Plasma, 13, p.022703 (2006). Cf. also D. Teychenné et al. Model and conservation laws... CEA Internal rep. R-6195 (2008).

[20] R. Sentis. Mathematical Models for Laser-Plasma Interaction, ESAIM-Math. Modelling Num. Analysis 39, p.275 (2005)

[21] R. Sentis. On the Boyd-Kadomtsev system for the three-wave coupling. Note C. R. Acad. Sci., Paris, Ser. I, Mathematique, 347, p. 933-938 (2009).

[22] S. Schochet, Fast singular limits of hyperbolic PDEs. J. Differential Equations, 114 (1994), 476-512.

[23] L. Tartar, An introduction to Navier-Stokes equation, Spinger, Berlin (2006). 